

\title{
A Prototype Model of Speculative Dynamics With Position-Based Trading
}

\author{
Reiner Franke* \\ Department of Economics \\ University of Kiel \\ Kiel, Germany
}

December 2007

\begin{abstract}
To avoid the indeterminate and generally unbounded positions of the agents in financial market models with order-based trading, the paper considers the alternative of position-based strategies. To this end it extracts a prototype model from the literature, with fundamentalists, chartists, and a risk-averse market maker. The deterministic formulation of the model leads to a neutral delaydifferential equation of the price, whose mathematical analysis is non-standard. The stability conditions are nevertheless quite analogous to the order-based Beja-Goldman model. The effects of parameter variations are also studied in a stochastic setting, where special emphasis is put on the misalignment between price and the time-varying fundamental value, and on the differential profits of fundamentalists and chartists.
\end{abstract}

JEL classification: C 15, D 84, G 12 .

Keywords: asset pricing; fundamentalists, chartists and market maker; misalignment; neutral delay-differential equation; random fixed point.

*Correspondence: franke@iksf .uni-bremen.de. Financial support from EU STREP ComplexMarkets, contract number 516446, is gratefully acknowledged. 


\section{Contents}

1 Introduction $\quad 1$

2 A direct inspiration from Beja and Goldman $\quad 3$

2.1 A recapitulation of the Beja-Goldman model . . . . . . . . . . . . . . . 3

2.2 A respecification of Beja-Goldman with position-based trading . . . . . . . . 6

$\begin{array}{lll}3 & \text { The deterministic position-based prototype model } & 9\end{array}$

3.1 Formulation of the model . . . . . . . . . . . . . . . . . . 9

3.2 Mathematical stability analysis . . . . . . . . . . . . . . . . 11

3.3 Numerical investigation of the stability frontiers . . . . . . . . . . . 15

3.4 Dynamic mechanisms over a cycle . . . . . . . . . . . . . . . 21

4 A stochastic version of the model $\quad 26$

4.1 Properties of a base scenario . . . . . . . . . . . . . . . . . 26

4.2 A stochastic equilibrium notion .................. 30

4.3 Parameter effects . . . . . . . . . . . . . . . . . 33

5 Conclusion $\quad 38$

$\begin{array}{ll}\text { A Appendix } & 39\end{array}$

A.1 Proof of Proposition $3 \ldots \ldots \ldots$. . . . . . . . . . . . . 39

A.2 Proof of Proposition 5 . . . . . . . . . . . . . . . . . 43

A.3 Recursive formulation of the stochastic PBT model . . . . . . . . . . . . . 44

A.4 Variations of parameters in the stochastic model . . . . . . . . . . . . 45

B References 46 


\section{Introduction}

In theoretical financial economics it is now widely acknowledged that a paradigm shift has taken place from the representative agent with his or her rational expectations towards a behavioural approach, in which markets are populated by heterogeneous and boundedly rational agents who use rule-of-thumb strategies. The success of a new paradigm is not the least dependent on neat and catchy model designs that capture some of the key ideas under discussion and show how the basic feedback mechanisms work out. An early prototype model of this kind has been put forward by Beja and Goldman (1980), who enthroned the market maker responsible for the gradual price adjustments together with the two archetypal groups of fundamentalists and chartists. The elementary setup and the intuitive outcome that fundamentalists tend to stabilize and chartists tend to destabilize the market had a great influence on the agent-based modelling that has begun in the 1990s. Whether the agents' asset demand is postulated directly or derived from expected utility maximization under constant absolute risk aversion (CARA), and whether the market impact of each of the speculative groups is constant or endogenously varying over time, in many of these models the original mechanisms of the Beja-Goldman model are still shining through. In one form or another, they remain important to an understanding of what is going on in different episodes of a model-generated price history.

Not only in the Beja-Goldman model but in the vast majority of agent-based asset pricing models, the strategies of the speculative traders are expressed in terms of their orders on the market. This is a problematic feature, although it is rarely seriously discussed. At least since Markowitz (1959) it is, first, a well established standard in portfolio choice theory that investors consider holdings rather than orders as the relevant object of their profit and risk evaluation. Second and more importantly, it is also well-known that in the orderbased framework the inventories of the agents and, in particular, the market maker are not anchored and so will be indeterminate. In a stochastic setting they may even easily become unbounded, which is certainly not compatible with the risk constraints of real traders.

There are a few models in the recent literature that adopt a different point of view and remove this inconsistency by letting the traders' strategies focus on their desired position. This branch of the literature is mainly connected with the name of Doyne Farmer: Farmer (2001) and Farmer and Joshi (2002), to cite the two papers with which we will be mostly 
concerned here. ${ }^{1}$ However, while the position-based models have examined a variety of single aspects so far, there is as yet no model formulation at a similar level of abstraction and insightfulness to the Beja-Goldman model. To prevent an anecdotal wilderness of bounded rationality as research proceeds further along these lines, it is the aim of the present paper to extract such a position-based prototype model from the two papers just mentioned. The outcome in the deterministic continuous-time framework will be a type of dynamic system that, as far as we know, shows up for the first time in economic modelling: a neutral delay-differential equation. It is more involved than a delay-differential equation, which is already of infinite dimension, since the right-hand side of the differential price equation contains not only the lagged level of the price but also its lagged derivative.

Despite its complexity it is possible to undertake a mathematical stability analysis. In fact, the resulting conditions on the parameters turn out to be analogous to the stability conditions in the two-dimensional Beja-Goldman model. Referring to a stylized periodic trajectory we will, however, also demonstrate that the cyclical mechanisms are different. Apart from that, a special feature of the model is an explicit risk aversion of the market maker, which has no counterpart in the order-based models.

In a second stage of the analysis, the model is reconsidered in a stochastic version, for which we employ the common assumption that the fundamental value follows a random walk. We briefly sketch the notion of a random fixed point and the pointwise convergence to it, which is a direct analogue of an attractive deterministic equilibrium. In a study of the effects of ceteris paribus variations of the model's parameters, the qualitative notion of stability is subsequently complemented by the quantitative concept of the degree of misalignment between price and fundamental value. In addition, we investigate the impact of a change in the parameters on the long-run profits of fundamentalists and chartists, where the prospects of the latter will be generally much better than in an order-based framework.

The paper proceeds as follows. In the next section we begin with a short recapitulation of the Beja-Goldman model and then try an immediate respecification of its fundamentalist

\footnotetext{
${ }^{1}$ Two references that take up Farmer's approach and develop it further in different directions are Carvalho (2001) and Pape (2007). An earlier paper that remains in a Beja-Goldman framework and incorporates the traders' inventory accumulation and cash flow into it is Sethi (1996). Some discussion of the market maker's profitability or inventory risk in an otherwise order-based framework can be found in Day (1997) and Westerhoff (2003).
} 
and chartist (flow) demand as position-based strategies. Since the resulting dynamics tend to be rather dull, Section 3 models the chartists in an alternative way, which, in the deterministic continuous-time framework, introduces lagged prices in their desired positions and thus a lagged price derivative in their market orders. This formulation yields our prototype model. The section contains the mathematical stability analysis, a numerical investigation of parameter variations and their impact on stability, and a study of the comovements of the variables on a stylized cyclical motion. Section 4 sets up the stochastic version, discusses the properties of a numerical base scenario and the random fixed point, and finally studies the effects of parameter variations. Section 5 concludes.

\section{A direct inspiration from Beja and Goldman}

\subsection{A recapitulation of the Beja-Goldman model}

Beja and Goldman (1980) distinguish three groups of participants in an asset market: two groups of speculators - fundamentalists and chartists - and a market maker. Fundamentalists have long time horizons and base their demand on the differences between the current price and the fundamental value. Even though they might expect the gap between the two prices to widen in the immediate future, they do not trade on these short-run expectations, choosing instead to place their bets on an eventual rapprochement. Chartists, on the other hand, neglect deviations of the price from the fundamental value and concentrate on shortrun changes. They use past movements of prices as indicators of market sentiments and extrapolate these into the next periods. In general, demand and supply of fundamentalists and chartists will not match the (fixed) supply of the asset. It is the primary function of the market maker to mediate transactions out of equilibrium. He executes all orders at the present price and accordingly decumulates (accumulates) inventories of the financial asset. His expectations and possible feedback effects from undesired inventories are ignored. So the demand of the market maker is treated as a residual and the only reaction considered here is the setting of a new price for the next trading round.

Let $p$ be the $\log$ price and $v$ the $\log$ of the fundamental value, on which the agents unanimously agree. As long as we are in a deterministic setting, here and in the following models, $v$ is supposed to remain constant. The fundamentalists specified by Beja and 
Goldman buy (sell) when $p$ is below (above) $v$. Chartist demand is characterized by a term $\pi$ that represents their perceptions of the current trend of prices. Both types of demand, denoted by $d^{f}$ and $d^{c}$, are specified in a linear manner. Introducing two positive coefficients $\phi$ and $\chi$, which may be taken as indicative of the aggressiveness of fundamentalists and chartists, respectively, or of their share of wealth in total speculative capital, we have

$$
\begin{aligned}
& d^{f}=\phi(v-p) \\
& d^{c}=\chi \pi
\end{aligned}
$$

To save an extra symbol for the supply of the asset, $d^{f}$ and $d^{c}$ may be interpreted as excess demand (in the verbal discussion we will also economize on the expression 'excess'). The dynamic relationships in the Beja-Goldman model are formulated in continuous time. Measuring the extent to which the market maker changes the price by a coefficient $\beta_{p}>0$, the evolution of prices is governed by the differential equation

$$
\dot{p}=\beta_{p}\left(d^{f}+d^{c}\right)
$$

The perception $\pi$ of the trend by the chartists is a predetermined variable, for which a simple adaptive updating rule is postulated. As the current 'trend' towards which the chartists seek to adjust their expectations is given by the instantaneous price changes, the rule is specified as

$$
\dot{\pi}=\beta_{\pi}(\dot{p}-\pi)
$$

where $\beta_{\pi}$ represents the speed at which these adjustments are carried out. This equation completes the description of the Beja-Goldman model. The four equations can be reduced to two linear differential equations in the price $p$ and the perceived trend $\pi$. They possess a unique equilibrium point $p=v$ and $\pi=0$, which (apart from a fluke) is either attractive or repelling. The stability condition can be formulated as follows.

\section{Proposition 1}

The equilibrium point of system (1) - (4) is asymptotically stable if $\chi<\phi / \beta_{\pi}+$ $1 / \beta_{p}$, and it is repelling if the inequality is reversed. A loss of stability under ceteris paribus variations of a parameter occurs by way of a Hopf bifurcation.

Proof: Substituting (1), (2) into (3) and the latter into (4), one gets the two-dimensional system 


$$
\begin{aligned}
\dot{p} & =\beta_{p}[\phi(v-p)+\chi \pi] \\
\dot{\pi} & =\beta_{\pi}\left[\beta_{p} \phi(v-p)+\left(\beta_{p} \chi-1\right) \pi\right]
\end{aligned}
$$

The determinant of its Jacobian is unambiguously positive, which rules out saddle point instability and zero eigen-values. The trace is negative if and only if the inequality stated in the proposition is satisfied.

$$
\text { q.e.d }
$$

The simple condition in the proposition allows one to characterize the parameters of the model as either stabilizing or destabilizing, meaning that sufficiently high values render a possibly unstable equilibrium stable or, respectively, they turn a possibly stable equilibrium into an unstable one. In this sense, the aggressiveness of fundamentalists $\phi$, or their weight in the market, is a stabilizing parameter, whereas a stronger weight $\chi$ of chartists is destabilizing. Presupposing $\chi>1 / \beta_{p}$, chartists can furthermore destabilize the equilibrium by a high adjustment speed $\beta_{\pi}$ in the updating of their expectations about future price changes. Effectively, $\beta_{\pi}$ is the rate at which old price changes are discounted. Thus, for a comparison with our main model later on, the instability feature may complementarily be also summarized by the statement that a short memory of chartists is destabilizing. A long memory (low $\beta_{\pi}$ ), on the other hand, can always ensure stability. Finally, sufficiently sluggish price adjustments $\beta_{p}$ of the market maker are stabilizing, and highly responsive adjustments are destabilizing if $\beta_{\pi} \chi>\phi$.

Because of the linearity of system (BG), the Hopf bifurcation in Proposition 1 is, of course, degenerate; periodic orbits only come into existence at the bifurcation value itself. We mention the Hopf bifurcation, here and in other propositions to follow, as a convenient short-cut which indicates that the positive and negative feedback mechanisms in the model provide considerable scope for cyclical behaviour. There are thus phases in the dynamics where the destabilizing forces (stemming from the chartists) dominate and drive the price away from the fundamental value, and other phases where the stabilizing forces (of the fundamentalists) gain momentum again and reverse this tendency. This interplay, whether explicitly referred to or not, has made the Beja-Goldman model an attractive basis for stochastic and deterministic extensions that give rise to richer dynamic phenomena. 


\subsection{A respecification of Beja-Goldman with position-based trad- ing}

In order to cope with the problem of indeterminate, unless unbounded, inventories in the Beja-Goldman model and to reconcile this model with the literature on position-based strategies, it is straightforward to redirect its focus from, so to speak, flows to stocks. In fact, the basic idea has already been put forward in a paper by Farmer (2001), though he does not mention the relationship or analogy to Beja-Goldman and is not concerned with a deterministic skeleton of the model variants he discusses.

To begin with, let $a^{f}, a^{c}, a^{m}$ be the asset positions of fundamentalists, chartists and the market maker. They can be positive and negative or, to be more precise, they may be conceived of as deviations from some fixed target positions. Three assumptions are then common in Farmer's work on position-based trading, which we here formulate in continuous time. The first two are only natural for a parsimonious modelling at the macroscopic level, the third one is introduced for a more specific reason.

(1) Dividends (in case of shares) or interest from foreign bonds (in case of a foreign exchange market), as well as the receipts from an alternative riskless asset are neglected as a source of reinvestment and accumulation of wealth. In other words, the market is supposed to be closed, so that the total number of assets is conserved: every time an agent buys an asset, another agent loses it. ${ }^{2}$ With the interpretation as deviations from a target, the agents' positions thus satisfy the following identity in every point in time,

$$
a^{f}+a^{c}+a^{m}=0
$$

(2) On the part of fundamentalists and chartists, one need not distinguish between desired and actual positions since the demand (in form of market orders) to which the desired positions give rise is always fulfilled by the market maker. As a consequence, the demand of the speculative agents and the rate of change in their positions coincide,

$$
d^{f}=\dot{a}^{f}, \quad d^{c}=\dot{a}^{c}
$$

(3) The third assumption will turn out to be a crucial mechanism to keep the market maker's inventory within bounds and even center it around its target. The essence of the

\footnotetext{
${ }^{2}$ In Farmer (2001) this seems to be tacitly understood; in Farmer and Joshi (2002) it is explicitly stated on p. 154 .
} 
price adjustment rule (3) is maintained, but an explicit risk aversion of the market maker is added. In Farmer's (2001, p. 66) specification, a negative (positive) position that has been accumulated prompts him to encourage selling (buying) by raising (lowering) the price more than usual. The risk that in this way impacts on the market is measured by a coefficient $\mu>0$. We also adopt Farmer's notation and replace the coefficient $\beta_{p}$ in (3) with $1 / \lambda$, which continues to be fixed over time. Here $\lambda$ is viewed as a scale factor and is referred to as the market's liquidity (or its depth), which nevertheless is assumed to be fixed. Upon closer scrutiny, this parameter has no time dimension and, nota bene, is not a speed of adjustment. $^{3}$ On the whole, price changes are now described by the equation

$$
\dot{p}=\left(d^{f}+d^{c}-\mu a^{m}\right) / \lambda
$$

According to what has just been said about $\lambda$, the coefficient $\mu$ has the same time dimension as the left-hand side of the equation: if the underlying time unit were shortened from one day to half a day, say, then $\mu$ would have to be reduced by fifty percent, too. It is worth mentioning that a term like $-\mu\left(a^{m}-x^{m}\right)$, where $x^{m}$ is the target level of the market maker's inventory, can be part of his optimal policy in a rigorous dynamic setting which, in particular, takes his uncertainty about the future arrival of tenders into account (Bradfield, 1979). ${ }^{4}$

Having thus established a basis for dealing with position-based strategies, we can change the Beja-Goldman model's type of demand from flows to stocks. Now fundamentalists do not buy if the market is undervalued but they hold the asset (take a long position), and if the price exceeds the fundamental value they do not sell but take a short position. Correspondingly so for the chartists if they perceive a positive or negative price trend, respectively. Formally it is postulated that the right-hand sides of eqs (1) and (2) determine the desired (and realized) positions of fundamentalists and chartists (rather than their market orders),

$$
a^{f}=\kappa_{f}(v-p)
$$

\footnotetext{
${ }^{3}$ See the derivation of the market impact function in Farmer and Joshi (2002, pp. 152f). Observe also that in connection with the coefficient $\beta_{p}$ in eq. (3) we have avoided the expression 'adjustment speed' (in contrast to Beja and Goldman, 1980, p. 237, themselves).

${ }^{4}$ The market structure in Bradfield's model is, however, more detailed than is usual in the present kind of modelling. A special result of his analysis is that, due to overnight costs, the coefficient $\mu$ depends on the time of the trading day: the later the time of the day, the less willing is the market maker to permit $a^{m}$ to diverge from $x^{m}$, so that transaction prices would be more variable at that time of the day.
} 


$$
\begin{aligned}
a^{c} & =\kappa_{c} \pi \\
\dot{\pi} & =\beta_{\pi}(\dot{p}-\pi)
\end{aligned}
$$

The last equation is eq. (4) from Beja-Goldman, which is here reproduced for convenience. The original coefficients $\phi$ and $\chi$ in (1) and (2) have been better replaced by $\kappa_{f}$ and $\kappa_{c}$ in the first two equations, since they have a different dimension in the new framework. Indeed, $\kappa_{f}$ and $\kappa_{c}$ are viewed as being proportional to the trading capital in the hand of the two groups of agents (cf. Farmer and Joshi, 2002, p. 158 and several times from p. 163 on). For easier erference, $\kappa_{f}$ and $\kappa_{c}$ are even identified with the trading capitals (which has motivated our choice of the Greek letter).

Equations (5) - (10) constitute a linear model where the formulation of demand in Beja-Goldman has been directly translated into position-based strategies. The model is again two-dimensional with the price $p$ and the perceived trend $\pi$ as state variables. As made explicit below, its reduced-form representation can indeed do without the positions, since the market maker's inventory in the price equation (7) is given by $a^{m}=-a^{f}-a^{c}$ and, using (8), (9), the latter two positions can be expressed in terms of $p$ and $\pi$. Proposition 2 collects the results of a stability analysis of this model.

\section{Proposition 2}

System (5)-(10) is well-defined and has a unique equilibrium point $p=v, \pi=0$ if $\lambda+\kappa_{f} \neq \beta_{\pi} \kappa_{c}$. Under this assumption the dynamics can be characterized as follows:

(a) The equilibrium is asymptotically stable if $\beta_{\pi} \kappa_{c} \leq \kappa_{f}$.

(b) Saddle point instability prevails if $\beta_{\pi} \kappa_{c}>\lambda+\kappa_{f}$.

(c) If $\kappa_{f}<\beta_{\pi} \kappa_{c}<\lambda+\kappa_{f}$, the equilibrium is asymptotically stable if $\mu<\mu^{c}:=$ $\beta_{\pi}\left(1+\alpha \beta_{\pi} \kappa_{c}\right) / \alpha\left(\beta_{\pi} \kappa_{c}-\kappa_{f}\right)$, and repelling if the inequality is reversed, where $\alpha:=1 /\left(\lambda+\kappa_{f}-\beta_{\pi} \kappa_{c}\right)>0$.

(d) In cases 1 and 3, the motions are monotonic (at least) if $\beta_{\pi} \geq \mu$.

The model shares with the Beja-Goldman model the property that fundamentalists are stabilizing (a sufficiently high weight $\kappa_{f}$ ) and chartists are destabilizing (large trading capital $\kappa_{c}$ or fast adjustments $\beta_{\pi}$ of their perceived trend). However, the model's scope for cyclical dynamics is rather limited, as shown by part $(b)$ and $(d)$ of the proposition. In contrast 
to what has been remarked on the Beja-Goldman at the end of the previous subsection, system (5)-(10) appears quite dull and not very promising for further extensions. The perhaps most interesting property is the feature stated in part $(c)$ that a high risk aversion of the market maker could destabilize the market, though a risk aversion exceeding the critical value $\mu^{c}$ would be more academic than real. The phenomenon can nevertheless be taken as a harbinger of a theme that will become more prominent in the next sections.

Proof: Using the definition of the coefficient $\alpha$ in part $(c)$ of the proposition, the two differential equations for $p$ and $\pi$ read,

$$
\begin{aligned}
& \dot{p}=f(p, \pi):=\alpha\left[\mu \kappa_{f}(v-p)-\kappa_{c}\left(\beta_{\pi}-\mu\right) \pi\right] \\
& \dot{\pi}=\beta_{\pi}[f(p, \pi)-\pi]
\end{aligned}
$$

The determinant of the Jacobian matrix $J$ is $\alpha \beta_{\pi} \mu \kappa_{f}$. Hence $\operatorname{det} J<0$ and the equilibrium is a saddle under the condition stated in the second part of the proposition. The rest of the proof supposes $\alpha>0$, so that the determinant is positive. The trace of the Jacobian can be written as trace $J=-\alpha \mu\left(\kappa_{f}-\beta_{\pi} \kappa_{c}\right)-\beta_{\pi}\left(1+\alpha \beta_{\pi} \kappa_{c}\right)$, which establishes part $(a)$. If $\kappa_{f}-\beta_{\pi} \kappa_{c}<0$, a negative trace is equivalent to the condition $\mu<\mu^{c}$ in part (c). To demonstrate the final part of the proposition, we can infer from an explicit calculation of the two eigen-values of the Jacobian that the system's trajectories are monotonic if $(\operatorname{trace} J)^{2}-$ $4 \operatorname{det} J=\left[\alpha \mu \kappa_{f}+\beta_{\pi}+\alpha \beta_{\pi} \kappa_{c}\left(\beta_{\pi}-\mu\right)\right]^{2}-4 \alpha \beta_{\pi} \mu \kappa_{f}$ is nonnegative. The supposition $\beta_{\pi}-\mu \geq 0$ ensures that this expression is not less than $\left(\alpha \mu \kappa_{f}+\beta_{\pi}\right)^{2}-4 \alpha \beta_{\pi} \mu \kappa_{f}=\left(\alpha \mu \kappa_{f}-\beta_{\pi}\right)^{2} \geq 0$.

q.e.d

\section{The deterministic position-based prototype model}

\subsection{Formulation of the model}

The main message of the previous section was a negative one: if one wants to set up a relatively simple model with position-based strategies and an interplay of negative and positive feedback effects that can provide broad scope for cyclical dynamics, then a direct respecification of the fundamentalist and chartist strategies from the Beja-Goldman model in terms of a demand for holding desired positions does not appear very fruitful. This result leads us to look for alternative strategies that could be more successful in this respect. A 
promising candidate are the trend followers that are put forward in a later paper by Farmer and Joshi (2002). These agents have a memory of $\theta$ time units, and their desired positions are proportional to the price change observed over this horizon. In their discrete-time and stochastic framework, Farmer and Joshi (pp. 154ff) establish that this strategy has the potential of amplifying the noise in the price and of inducing oscillations at frequencies that are related to the length of the memory $\theta$.

Regarding the market maker in this paper, Farmer and Joshi make no mentioning of his possible risk aversion. To deal with the problem that prices and fundamental values may easily drift apart, they rather assume a large number of trend followers and fundamentalists with individual trading capitals and, in addition, individual thresholds for entering and exiting a position. The numerical simulation experiments demonstrate that this strong heterogeneity can indeed achieve a cointegration of price and value (if the parameter choices take care of certain trade-off effects; see Farmer and Joshi, 2002, p. 167, fn 10). However, the very construction and purpose of this model make it meaningless to ask for a lowdimensional deterministic skeleton that could be studied on its own.

In order to design such a prototype model, we extract two central ideas from the concepts presented so far and combine them with the position-based fundamentalists from above. First, we use Farmer and Joshi's (2002) specification of trend followers as our chartist strategy. This element should give sufficient prospects for dynamic feedback mechanisms generating oscillatory behaviour. Second, we reintroduce the risk aversion of the market maker from Farmer (2001), which by keeping his inventory within bounds should also be able to prevent persistent misalignment. Our analysis begins with the deterministic and continuous-time formulation of this model mix.

Since with the introduction of the time lag $\theta>0$ chartists base their decisions on prices in two different moments in time, the time index can no longer be dropped. The (desired and realized) positions of fundamentalists and chartists are given by

$$
\begin{aligned}
a^{f}(t) & =\kappa_{f}[v-p(t)] \\
a^{c}(t) & =\kappa_{c}[p(t)-p(t-\theta)]
\end{aligned}
$$

Equation (11) reiterates (8), and (12) corresponds to eq. (8) in Farmer and Joshi (2002, p. 154). 
The evolution of prices continues to be governed by (7). By plugging (11), (12) into eq. (6) for the market orders, solving identity (5) for the market maker's position $a^{m}(t)$, and substituting the resulting expressions in the price equation (7), the market dynamics can be reduced to the following differential equation:

$$
\dot{p}(t)=\frac{\mu \kappa_{f}[v-p(t)]+\mu \kappa_{c}[p(t)-p(t-\theta)]-\kappa_{c} \dot{p}(t-\theta)}{\lambda+\kappa_{f}-\kappa_{c}}
$$

The acronym PBT serves to indicate that the equation summarizes a model with positionbased trading, the one that we will be concerned with in the rest of the paper.

Given $\mu>0$, the model has a unique equilibrium price $p=v$, which implies overall zero positions $a^{f}=a^{c}=a^{m}=0$ in that state. Note that without the market maker's risk aversion, when $\mu=0$, the equilibrium price would be indeterminate and so would be the positions of fundamentalists and the market maker. Of course, this is the reason why prices do not track values in the simpler versions of Farmer and Joshi's model (see, in particular, Farmer and Joshi, 2002, p. 159).

\subsection{Mathematical stability analysis}

The reduced form (PBT) of the price dynamics is of a remarkable type. The lagged price $p(t-\theta)$ on the right-hand side makes it a (linear) delay differential equation (or a differential equation of retarded type), while the lagged derivative $\dot{p}(t-\theta)$ makes it a so-called neutral delay differential equation. Beginning with the pioneering work of Kalecki (1935) and Goodwin (1951), delay differential equations are occasionally encountered in the (nonorthodox) modelling of dynamic economic systems. We do not know, however, of one single example of a neutral delay differential equation in that field. Moreover, even in the more advanced textbooks on economic dynamics this type of equation is at best mentioned in an introductory classification, but any further treatment of it is lacking (e.g., in Gandolfo, 1997, pp.550f). Since the standard literature provides no mathematical theorems that could be utilized to inquire into the stability of a neutral delay differential equation like (PBT), we have to collect several different results from a (small) number of more specialized mathematical papers, which are even of quite recent dates. The following analysis may therefore also be of some independent interest to economists working with dynamic systems. 
Differential equations with delays are of infinite dimension. This is reflected by the fact that the initial conditions to start a trajectory require the specification, not of a vector with finitely many components, but of an entire time path of the state variable(s). The initial price path in the present model has also to be taken into account by the precise definition of stability. ${ }^{5}$ Thus, to be perfectly clear, consider a starting time $t=0$, let $p^{o}=p^{o}(t)$ be a differentiable function on the interval $[-\theta, 0]$ that specifies the initial condition for system $(\mathrm{PBT})$, and denote the corresponding solution of $(\mathrm{PBT})$ as $p=p\left(t ; p^{o}(\cdot)\right)$. The equilibrium point $v$ is called stable if for any $\varepsilon>0$ there exists a number $\delta>0$ such that every initial time path $p^{o}(\cdot)$ with $\left|p^{o}(t)-v\right|<\delta$ for $t \in[-\theta, 0]$ gives rise to a trajectory that satisfies $\left|p\left(t ; p^{o}(\cdot)\right)-v\right|<\varepsilon$ for all $t>0$. If in addition $p\left(t ; p^{o}(\cdot)\right) \rightarrow v$ as $t \rightarrow \infty$, the equilibrium is called asymptotically stable. Synonymously, also system (PBT) itself my be called (asymptotically) stable. It goes without saying that because everything is linear here, local stability amounts to global stability.

The dynamic properties of the system continue to be determined by the roots of the characteristic equation. However, what is true for delay differential equations is all the more true for neutral equations: rather than a polynomial the characteristic equation becomes a transcendental equation, which has a countably infinite number of roots (cf. Gandolfo, 1997, p. 551). The whole stability analysis rests on the conclusions that can be drawn for the real parts of these roots. The results that we are thus able to derive are summarized in the next proposition. Its proof is given in the appendix.

\section{Proposition 3}

Assume $\kappa_{c}<\lambda+\kappa_{f}$. Then the following holds:

(a) If $\kappa_{c}<\kappa_{f} / 2$, system (PBT) is asymptotically stable.

(b) If $\kappa_{c}>\left(\lambda+\kappa_{f}\right) / 2$, system (PBT) is unstable.

(c) A loss of stability under ceteris paribus variations of a parameter occurs by way of a Hopf bifurcation.

(d) If $\kappa_{f} / 2<\kappa_{c}<\left(\lambda+\kappa_{f}\right) / 2$, there exists a bifurcation value $\theta^{\star}>0$ of the $\operatorname{lag} \theta$ such that $(P B T)$ is asymptotically stable if $0<\theta<\theta^{\star}$, and unstable if $\theta>\theta^{\star}$. For short lags, the equilibrium is generically approached in a

\footnotetext{
${ }^{5}$ See, e.g., Han (2001, p. 966) for an explicit statement of the following definition.
} 
monotonic manner. For values of $\theta$ in a certain (possibly wide) neighbourhood of $\theta^{\star}$, the motions of (PBT) are generically of a cyclical nature. Close to $\theta^{\star}$, their period is $T \approx 2 \pi / \nu^{\star}>2 \theta^{\star}$, where

$$
\nu^{\star}:=\sqrt{\frac{\mu^{2} \kappa_{f}\left(2 \kappa_{c}-\kappa_{f}\right)}{\left(\lambda+\kappa_{f}\right)\left(\lambda+\kappa_{f}-2 \kappa_{c}\right)}}
$$

The stability conditions bear a close resemblance to those of Proposition 1 for the BejaGoldman (BG) model. In particular, fundamentalists and chartists maintain their basic roles. Thus, a sufficiently large trading capital $\kappa_{f}$ of the fundamentalist trading group can always stabilize the equilibrium, just like the corresponding parameter $\phi$ in (BG). Chartists, on the other hand, destabilize the market if their trading capital $\kappa_{c}$ is large, which parameter is analogous to the chartist aggressiveness $\chi$ in $(B G)$. As far as the responsiveness of prices is concerned, the counterpart of a high market liquidity $\lambda$ in (PBT) is a high value of the reciprocal $1 / \beta_{p}$ of the price adjustment parameter in $(\mathrm{BG})$, and both have a stabilizing effect on the dynamics. A further common feature of the two models is their potential to generate cyclical motions of the price. Proposition $3(d)$ asserts that these cycles are essential in (PBT), in the sense that their period is at least twice as long as the memory of the chartists.

The memory of chartists in (PBT) and (BG) cannot be directly compared. In the discussion of Proposition 1 a long memory has been identified with slow adjustments of the perceived trend and so with low values of the adjustment speed $\beta_{\pi}$. With this interpretation a long memory was stabilizing. In (PBT), a long memory is represented by a long time horizon $\theta$, which according to Proposition $3(d)$ is destabilizing if $\kappa_{c}$ is contained in the intermediate interval $\left(\kappa_{f} / 2,\left(\lambda+\kappa_{f}\right) / 2\right)$. This characterization would, however, be misleading since longer lags $\theta$ tend to increase the difference between the current and the lagged price $p(t-\theta)$. If one wants to control this effect it would be more appropriate to specify eq. (12) as

$$
a^{c}(t)=\tilde{\kappa}_{c}[p(t)-p(t-\theta)] / \theta
$$

The coefficient $\kappa_{c}$ in eq. (PBT) would then read $\tilde{\kappa}_{c} / \theta$, and this expression remains no longer fixed as $\theta$ varies. With this observation we can more specifically conclude that, with a given value of $\tilde{\kappa}_{c}$, a sufficient increase of $\theta$ would eventually let the term $\tilde{\kappa}_{c} / \theta$ fall below the critical value $\kappa_{f} / 2$ of Proposition 3(a). Conversely, if $\theta$ approaches zero, the same term 
would eventually exceed the threshold stated in part $(b)$ of the proposition. Hence we obtain an additional stability result.

\section{Proposition 4}

Replace eq. (12) with eq. (13) in the PBT model. Then the equilibrium is asymptotically stable if the lag $\theta$ is large enough, and unstable if it is sufficiently short.

Referring more loosely to the memory of chartists, we can now say that in both the BG model and the reformulated PBT model a long memory is stabilizing and a short memory is destabilizing.

The overall conclusion from the propositions is that (PBT) as a stylized model with position-based fundamentalist and chartist traders has very similar dynamic properties to the Beja-Goldman model with its order-based strategies. In a form that emphasizes the analogies between (PBT) and (BG), the parameter effects on the stability of the equilibrium point are succinctly summarized in Table 1. The present model should thus be a good basis for more ambitious small-scale modelling attempts within a position-based framework, from which one could expect comparable results, at least numerically, to what has been obtained in the order-based framework.

\begin{tabular}{llllll}
\hline & weight $\mathrm{F}$ & weight $\mathrm{C}$ & memory C & liquidity & risk aversion MM \\
\hline PBT : & $\mathrm{S}\left(\kappa_{f}\right)$ & $\mathrm{D}\left(\kappa_{c}\right)$ & $\mathrm{S}(\theta)$ & $\mathrm{S}(\lambda)$ & $\mathrm{D}(\mu)$ \\
$\mathrm{BG}:$ & $\mathrm{S}(\phi)$ & $\mathrm{D}(\chi)$ & $\mathrm{S}\left(1 / \beta_{\pi}\right)$ & $\mathrm{S}\left(1 / \beta_{p}\right)$ & - \\
\hline
\end{tabular}

Table 1: Parameter effects on stability in (PBT) and (BG).

Note: S (D) means that a sufficiently large increase of the parameter (de)stabilizes the equilibrium. Regarding $\theta$, the specification of eq. (13) with a fixed value of $\tilde{\kappa}_{c}$ is assumed.

There is one parameter left in the PBT model that has no counterpart in (BG), namely, the risk aversion $\mu$ of the market maker. Returning to the original formulation of the model with eq. (12) and concentrating the interest on the inconclusive parameter interval for $\kappa_{c}$, where stability as well as instability may prevail, it is relatively easy to get 
a definite characterization of the coefficient $\mu$, too. As shown in Appendix A.2, the proof of the next proposition is almost a by-product of the proof of Proposition $3(d)$.

\section{Proposition 5}

Suppose $\kappa_{f} / 2<\kappa_{c}<\left(\lambda+\kappa_{f}\right) / 2$ in eq. $(P B T)$, consider two values $\mu_{1}, \mu_{2}$ of the market maker's risk aversion, and, fixing the other parameters, let $\theta_{1}^{\star}, \theta_{2}^{\star}$ be the corresponding bifurcation values of the lag $\theta$. Then $\theta_{2}^{\star}=\mu_{1} \theta_{1}^{\star} / \mu_{2}$.

The consequence of the proposition is immediate. Fix all parameters except $\mu$ and start from some intermediate value $\mu_{1}$ with associated bifurcation value $\theta_{1}^{\star}$, for which we may assume that it entails stability, $\theta<\theta_{1}^{\star}$. Then the proposition says that the bifurcation lag $\theta^{\star}=\theta^{\star}(\mu)$ is reduced as $\mu$ begins to rise. If $\mu$ rises far enough, the model's lag $\theta$ will no longer be contained in the shrinking stability interval, $\theta>\theta^{\star}(\mu)$, and the equilibrium becomes unstable. Hence, under the given condition on $\kappa_{f}, \kappa_{c}$ and $\lambda$, a higher risk aversion of the market maker tends to be destabilizing. Recalling the destabilizing risk aversion found in Proposition 2(c) for our first position-based model, this result now turns out to be not so atypical after all.

The mechanism also works in the opposite direction. If initially $\mu=\mu_{1}$ gives rise to instability, $\theta>\theta_{1}^{\star}$, then a sufficient lowering of $\mu$ lets $\theta^{\star}(\mu)$ increase above $\theta$ and so renders the equilibrium stable. In this sense a low risk aversion of the market maker may be called stabilizing. It has, however, to be taken into account that this would also slow

down the speed at which the market maker's inventory approaches its target level. One easily finds numerical examples where convergence toward the equilibrium takes so long that classifying this system as stable would rather be misleading. In particular, already weak random perturbations would suffice to completely blur any tendency of the price to return to the fundamental value.

\subsection{Numerical investigation of the stability frontiers}

The mathematical stability analysis allowed us to state that sufficiently low values of a parameter entail stability of the equilibrium and/or sufficiently large values of this parameter entail its instability; or the other way around. Hence a ceteris paribus variation of the parameter renders a stable equilibrium unstable, or vice versa. For example, with respect 
to the chartist trading capital $\kappa_{c}$ Proposition 3 tells us that such a bifurcation occurs somewhere in the interval $\left(\kappa_{f} / 2,\left(\lambda+\kappa_{f}\right) / 2\right)$. Likewise, if $2 \kappa_{c}>\lambda$ then a rise of $\kappa_{f}$ beginning at zero stabilizes the originally unstable dynamics somewhere in the interval $\left(2 \kappa_{c}-\lambda, 2 \kappa_{c}\right)$. A question that the mathematical analysis could not answer, however, is whether these bifurcations are unique, i.e., whether a reswitching of stability or instability can be ruled out. Except for the lag parameter $\theta$, for which a unique bifurcation is ensured by Proposition $3(d)$, we have here to resort to numerical calculations.

Although the mathematical theory of neutral delay differential equations is quite involved, numerical bifurcations can be studied by checking a surprisingly simple criterion. To this end, let $\eta$ stand for one of the parameters $\kappa_{f}, \kappa_{c}, \lambda$ or $\mu ; \eta$ is supposed to vary and the other parameters remain fixed. Equation (21) in Appendix A.1 specifies $a_{0}, b_{0}, b_{1}$ as composite terms of the four coefficients, so that for the present purpose we may write them as $a_{0}=a_{0}(\eta)$, etc. In addition, we refer to $\nu^{\star}=\nu^{\star}(\eta)$ from the formulation of Proposition 3. Then, consider the functional expression

$$
h(\theta, \eta):=a_{0}(\eta)+b_{0}(\eta) \cos \left[\nu^{\star}(\eta) \theta\right]+b_{1}(\eta) \nu^{\star}(\eta) \sin \left[\nu^{\star}(\eta) \theta\right]
$$

which is at the heart of the bifurcation analysis. From eq. (24) in the appendix and the subsequent arguments it can be inferred that an admissible value $\eta^{\star}$ is a bifurcation value if, and only if, it satisfies

$$
h\left(\theta, \eta^{\star}\right)=0 \quad \text { and } \quad \nu^{\star}\left(\eta^{\star}\right) \theta<\pi
$$

Numerical studies of (15) are somewhat facilitated by the feature that, for example, $\lambda$ can serve as a scaling parameter in (PBT). In fact, it is obvious that multiplying $\lambda$ as well as $\kappa_{f}$ and $\kappa_{c}$ by the same factor leaves the differential equation completely unaffected. So when we fix the parameters in a numerical scenario, let us adopt the normalization $\lambda=1$.

An investigation of eq. (15) over a wide range of the other parameters led us to the conclusion that unique bifurcation values can be considered to be the rule. There are exceptions to this principle, but it requires some effort to find them. ${ }^{6}$ The bold (blue) line in Figure 1 presents one such counterexample for the trading capital $\kappa_{f}$ of the fundamentalists

\footnotetext{
${ }^{6}$ The function $\eta \mapsto h(\theta, \eta)$ has infinitely many roots if the variations of $\eta$ let the expression $\left(\lambda+\kappa_{f}-2 \kappa_{c}\right)$ converge to zero. However, in these cases the term $\nu^{\star}(\eta)$ usually gets so large that it violates the second constraint in (15). The task is to find an 'unusual' case of these oscillations where a root $\eta^{\star}$ is already so far away from the boundary that $\nu^{\star}\left(\eta^{\star}\right) \theta<\pi$.
} 
as the bifurcation parameter. Given the coefficients reported in the note added to Figure 1 and, in particular, $\kappa_{c}=0.480$, stability prevails for $0<\kappa_{f}<0.00874$ and for $\kappa_{f}>0.08227$, whereas the equilibrium is unstable for values of $\kappa_{f}$ in the intermediate interval.

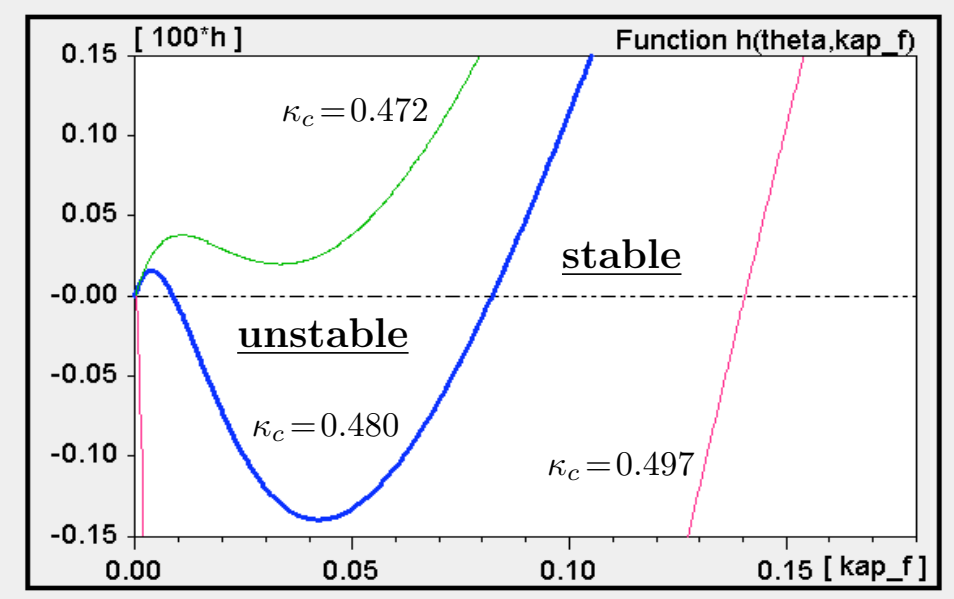

Figure 1: Reswitching of stability under variations of $\kappa_{f}$.

Note: The graph of the function $\kappa_{f} \mapsto h\left(\theta, \kappa_{f}\right)$ specified in (15), given $\lambda=1$, $\mu=0.05, \theta=40$ and three values of $\kappa_{c}$ as indicated. Positive (negative) values of $h$ entail stability (instability).

The shape of the function $\kappa_{f} \mapsto h\left(\theta, \kappa_{f}\right)$ under $\kappa_{c}=0.480$ illustrates the difficulties that one will have in a general analytical treatment of the uniqueness conjecture. At the same time, the diagram also demonstrates the limited significance of multiple bifurcations of a parameter. First, note the relatively narrow and low range of $\kappa_{f}$ over which the reswitching is obtained (this will become even more evident in Figure 2 below). Second, the reswitching phenomenon does not appear to be very robust, either, since already small changes in the (exogenous) parameter $\kappa_{c}$, in either direction, are sufficient for the multiple roots of the function $h(\theta, \cdot)$ to disappear. Thus, at $\kappa_{c}=0.472$ the function $h$ has a similar qualitative shape, but the decline after the initial rise is no longer strong enough to reach the zero line; see the thin (green) line in the upper half of Figure 1. Hence the equilibrium is here stable for all $\kappa_{f}>0$. At a higher level of the chartist trading capital such as 
$\kappa_{c}=0.497$, the function decreases steeply right from the beginning and, after increasing again, cuts the zero line only once (at $\left.\kappa_{f}=0.14044\right)$.

Regarding multiple bifurcations of the parameters as rather exceptional cases sharpens the stability statements of the mathematical propositions. Accordingly, the trading capital $\kappa_{f}$ of fundamentalists and market liquidity $\lambda$ can be characterized as being (almost) unambiguously stabilizing. Which means that if the equilibrium is not yet stable, then it can be made stable by a sufficient increase of $\kappa_{f}$ or $\lambda$-and stability is preserved if these parameters are further increased. Conversely, if the equilibrium is stable then an arbitrary decline of $\kappa_{f}$ below a certain threshold destabilizes it. Low values of $\lambda$ have the same effect unless $\kappa_{c} \leq \kappa_{f} / 2$. In an analogous sense, the trading capital $\kappa_{c}$ of chartists and the risk aversion of the market maker $\mu$ are (almost) unambiguously destabilizing. The close correspondence to the stability results in the Beja-Goldman model, where multiple bifurcations are ruled out, has already been pointed out above in a comment on Proposition 3.

A summary of the stabilizing or destabilizing properties of the parameters can be made more vivid by computing the stability frontiers in a paremeter plane. Most expedient for this are the trading capitals $\kappa_{f}$ and $\kappa_{c}$ of the two speculative groups, where now a greater range of the two coefficients than in Figure 1 should be considered. To set up a benchmark of the other three parameters, we maintain the scaling $\lambda=1$ for market liquidity and the market maker's risk aversion $\mu=0.05$, and reduce the memory of chartists by half, i.e., we put $\theta=20$. The stability frontier in the $\left(\kappa_{f}, \kappa_{c}\right)$ plane to which these coefficients give rise is shown in the upper-left panel in Figure 2; it separates the pairs entailing stability (in the dotted area) from those entailing instability. Given that the functional expression $h$ in eq. (14) is fairly nonlinear in $\kappa_{c}$ and $\kappa_{f}$, it is remarkable that the stability frontier is an almost perfect straight line.

Proposition $3(d)$ has established that an originally stable equilibrium is destabilized by sufficiently long lags $\theta$, provided $\kappa_{c}$ is contained in the intermediate interval $\left(\kappa_{f} / 2,\left(\lambda+\kappa_{f}\right) / 2\right)$ and eq. (12) is retained (and not replaced with (13), which is the case covered by Proposition 4). The upper-right panel in Figure 2 considers the destabilizing effect from a different point of view. It shows that an increase of the lag $\theta$ from 20 to 40 causes the stability region to shrink, although the reduction is rather limited. Note the tiny 'hook' at the lower end of the stability frontier, which owing to the large scale of $\kappa_{f}$ and $\kappa_{c}$ is hardly recognizable: for 


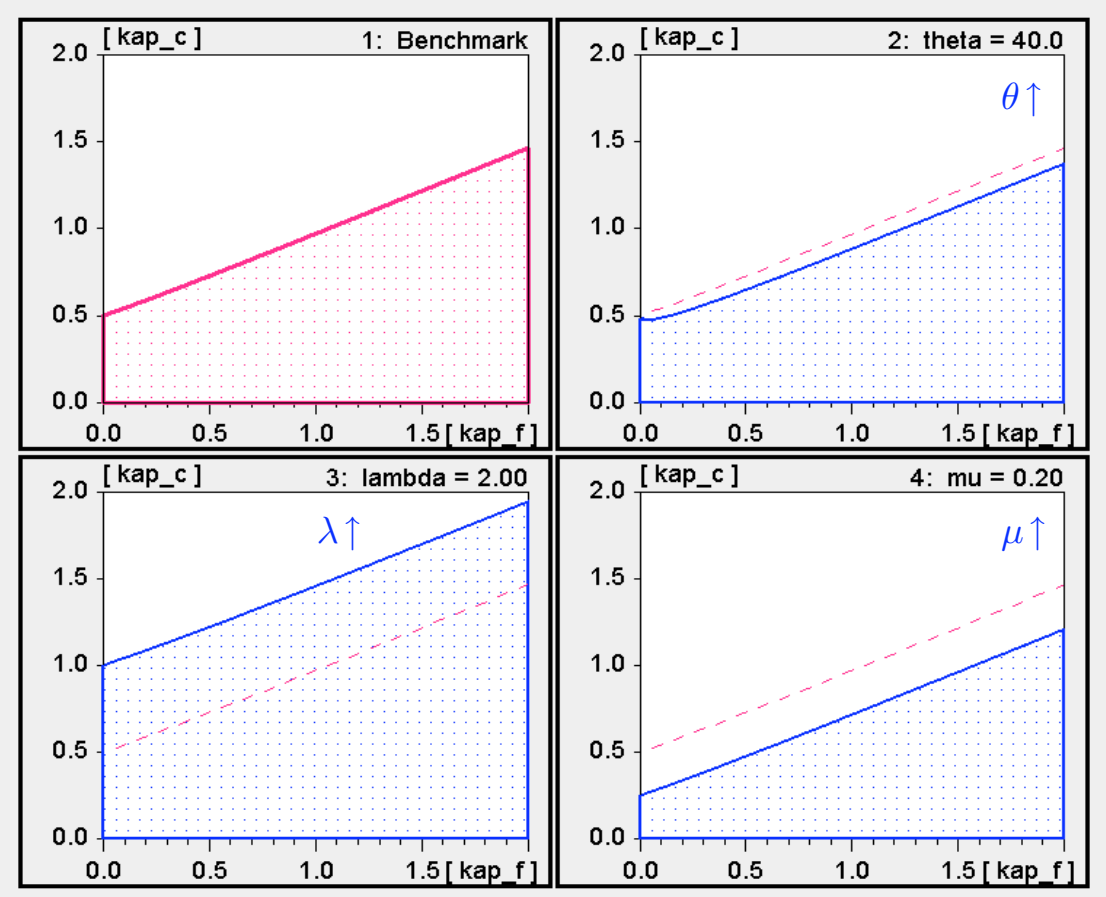

Figure 2: Stability frontier of $(\mathrm{PBT})$ in the $\left(\kappa_{f}, \kappa_{c}\right)$ parameter plane.

Note: The dotted area is the stability region. The (red) dashed lines reproduce the stability frontier of the benchmark case in the first panel, which is based on $\lambda=1$ $\mu=0.05, \theta=20$.

$\kappa_{c}$ slightly less than 0.50 we have here the stability reswitching of $\kappa_{f}$ from Figure 1 (which had $\theta=40$ underlying).

The other two panels in Figure 2 demonstrate the stabilizing effect when market liquidity is increased from 1 to $\lambda=2$, and the destabilizing effect when the market maker's risk aversion is increased from 0.05 to $\mu=0.20$. Again there are no ambiguities in the relocation of the stability frontier, practically it is even a parallel upward and downward shift, respectively. In sum, Figure 2 is a representative illustration of the fact that the parameter effects on stability are of an amazingly regular nature.

A special feature of the model is that we have an explicit expression for the cycle period $T$ induced by the parameters on the stability frontier, $T=2 \pi / \nu^{\star}$ according to Proposition $3(d)$. While the mathematical analysis has made sure that the period is significantly longer than the time horizon $\theta$ of the chartists, $T>2 \theta$, it does not tell us by how much $T$ exceeds this lower boundary. For this and to get an impression of how $T$ varies with the parameters 
of the model, we better resort to specific numerical values and make use of the stability frontiers already computed.

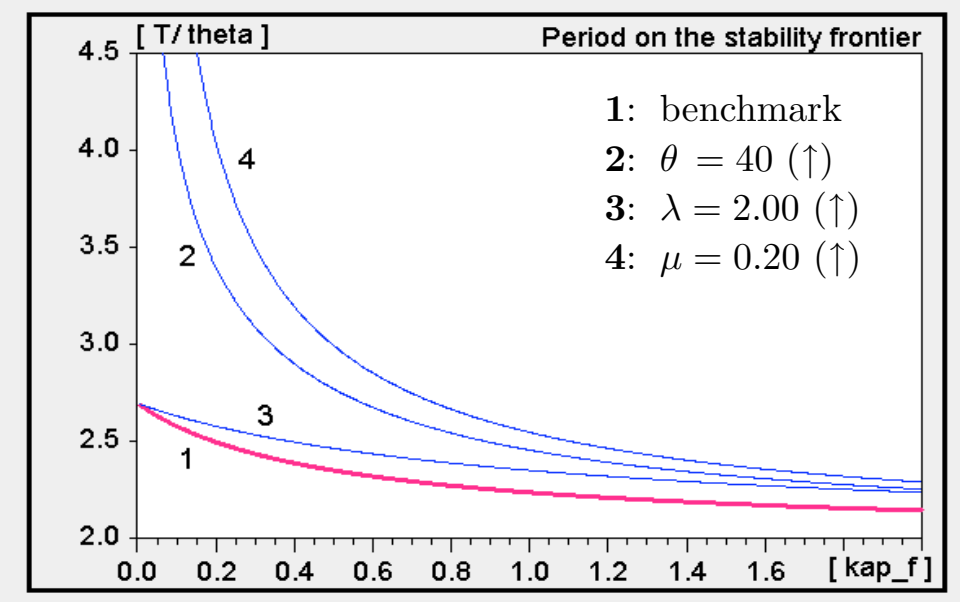

Figure 3: Period $T$ on the four stability frontiers from Figure 2.

Figure 3 depicts on the horizontal axis the same values of $\kappa_{f}$ as in Figure 2. It takes the parameters underlying the latter diagram, computes the bifurcation values of $\kappa_{c}$ given $\kappa_{f}$, plugs all these coefficients into the formula for $\nu^{\star}$, and then draws $T=2 \pi / \nu^{\star}$ as a function of $\kappa_{f}$. Numbers $1 \ldots 4$ in Figure 3 indicate the panels in Figure 2 from which the functions are thus obtained. Since the second panel has examined a different lag $\theta$ from the other panels, Figure 3 plots the fraction $T / \theta$.

One property that the four functions have in common is that they decline with $\kappa_{f}$. The initial level and the slope of the functions are, however, quite distinct. Our benchmark case (the bold line) implies relatively short oscillations. They increase, though not very much, for deeper markets (line 3 brought about by a rise in $\lambda$ ). However, if a model builder requires a period three times or more as long as the memory $\theta$, then he or she will consider the option of increasing $\theta$ itself or the risk aversion $\mu$ of the market maker, where at the same time the trading capital of fundamentalists and chartists should be rather limited (in relation to the fixed market liquidity $\lambda$ ). 
Regarding the cycle prolonging effects of an increase in $\mu$ it is interesting to quote a remark by Farmer on the market maker, although he made it in a stochastic context with no chartists: "Once the market maker acquires a position, because of her risk aversion, she has to get rid of it. By selling a fraction $\beta$ [our coefficient $\mu$ ] at each time step, she will unload the position a bit at a time. This behavior causes a trend in prices. Any risk-averse behavior on the part of the market maker will result in a temporal structure of some sort in prices." (Farmer, 2001, p.67). It is noteworthy that this tendency still prevails when we restrict our attention to the periodic orbits generated by the bifurcation values of the parameters.

One point following from these considerations is that temporal structure in prices creates an opportunity for technical traders (ibid., p.68). This observation motivates us to have now a look at the time series properties of the model and, in particular, the implied performance of the agents.

\subsection{Dynamic mechanisms over a cycle}

While cycle generating feedbacks in models with order-based strategies are well understood, these aspects are still largely neglected in the position-based systems. For this reason we want to study a stylized cyclical motion of (PBT), without any random noise and unaffected by nonlinear amendments like thresholds and flexible ceilings and floors. It will also be illuminating to compare the cyclical patterns of the variables with those arising from the order-based Beja-Goldman model. The parameters that we employ to obtain periodic orbits of these linear systems read as follows: ${ }^{7}$

$$
\begin{aligned}
& \mathrm{BG}: \phi=0.20 \quad \chi=2.000 \quad \beta_{p}=1 \quad \beta_{\pi}=0.20 \\
& \text { PBT: } \phi=0.20 \quad \kappa_{c}=0.616 \quad \lambda=1 \quad \theta=20 \quad \mu=0.05
\end{aligned}
$$

The results for the two models are contrasted in Figure 4, where the BG model is shown in the upper part and the PBT model in the lower part. Let us begin with system (BG), at a point in time when the price is rising and just passes the fundamental value, which is normalized at zero. This happens at $t=39.50$ in the top panel of the diagram. Since the

\footnotetext{
${ }^{7}(\mathrm{PBT})$ is discretized with $\Delta t=1$, which is not too large given the lag $\theta=20$. The bifurcation value $\kappa_{c}$ given the other parameters is slightly higher (not lower!) than it would be in continuous time. For (BG) the step size is so small that the continuous-time and discrete-time bifurcation values of $\kappa_{c}$ are practically the same.
} 
demand of fundamentalists is a negative mirror image of the price movements, the price increases are here solely due to the demand of the chartists. The latter is positive since the price has already been rising for a while, such that the perceived trend of the chartists has become positive in the meantime.
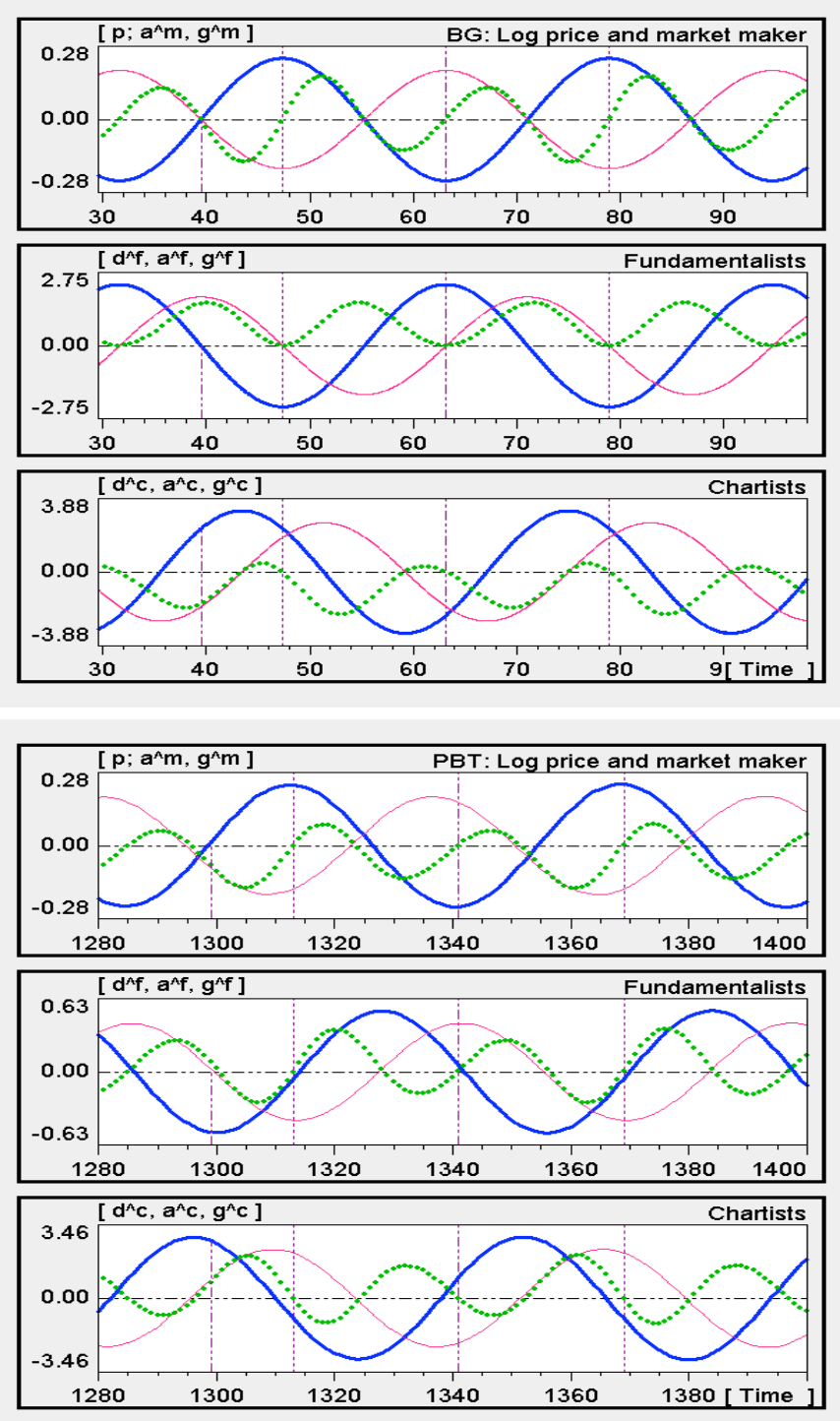

Figure 4: Price series and the agents' characteristics in (BG) and (PBT).

Note: The bold (blue) line in the top panel of (BG) and (PBT), respectively, is the $\log$ price, the bold lines in the other panels depict market orders, or demands, $d^{f}$ and $d^{c}$. The thin solid (red) lines are the positions $a^{m}, a^{f}, a^{c}$, respectively, the dotted (green) lines are the profit flows, or capital gains, $g^{m}, g^{f}, g^{c}$. Market orders and profits are rescaled to fit into the diagrams. 
A central feature of the cycle generating mechanism in the BG model is the fact that the more the price increases, the stronger becomes the negative impact on the part of the fundamentalists. The ensuing slow down in the price increases works to the effect that eventually the rising trend as it is perceived by the chartists, $\pi$, comes to a halt and then begins to decline. Since this occurs at a relatively high level of $\pi$, chartist demand still exceeds fundamentalist demand for some time. At $t=47.38$, the difference between the two narrows down to zero, so that $\dot{p}=0$ and the price reaches an upper turning point. From then on $p$ is falling; the decline in $\pi$ accelerates and $d^{c}$ decreases faster as $d^{f}$ now begins to increase. Hence the negative demand of the fundamentalists dominates the (here still positive) demand of the chartists. As the decline in the price goes on, the dynamics enters the stage with which we have begun, now with signs reversed.

Before discussing the implied positions and profits, let us turn to the lower part of Figure 4 with the dynamics of the PBT model. The initial conditions have been chosen such that with \pm 0.23 the price oscillations exhibit the same amplitude as in the BG model. We consider the same phase of a cycle as before, beginning with the upward movement of the price when it just crosses the zero line at $t=1298$. For the fundamentalists it is now the position $a^{f}$ rather than the demand that is a negative mirror image of the price series; see the thin solid (red) line in the middle panel. Since $a^{f}$ is falling at $t=1298$, fundamentalist demand $d^{f}=\dot{a}^{f}$ is initially negative. At that time it is even close to its trough value over the entire cycle. Note, however, that the amplitude of $d^{f}$ in (PBT) is more than four times lower than in $(\mathrm{BG}), \pm 0.52$ versus \pm 2.29 .

In order for the price to increase at $t=1298$, chartist demand $d^{c}$ must dominate $d^{f}$, which can be seen in the bottom panel. The reason for the relatively high demand is not the positive position $a^{c}$, which is proportional to the slope in the log price $p(1298)-p(1278)$, but the fact that this slope is presently rising, so that $a^{c}$ is rising and thus $d^{c}=\dot{a}^{c}>0$. In addition the negative inventory $a^{m}$ of the market maker has to be taken into account, which via the correction term $-\mu a^{m}>0$ in the price impact function (7) reinforces the rise in $p$.

In the next few periods there are two features that, taken on their own, would accelerate the price increase. First, the increase in the market maker's short position, i.e., the ongoing decline in $a^{m}$. Second, the position of fundamentalists declines, too, but at a lesser speed. Hence, while $d^{f}=\dot{a}^{f}<0$, the influence of the supply from this side of the 
market tends to weaken. Chartist demand, however, counteracts these forces. Although chartists continue to build up long positions, they do this more and more slowly. In fact, their position reaches the highest level at $t=1310$, afterwards $\dot{a}^{c}<0$ and chartists turn into net suppliers of the asset.

The price peaks three periods later, at $t=1313$. Its increase over this short interval where both $d^{c}$ and $d^{f}$ are negative is due to the market maker, who with his accumulated short position still feels it necessary to give some incentive to the agents to sell the asset to him.

The beginning downturn of the price gains momentum through the increasing desire of the chartists to cut down on their long position, though for a while they keep on wanting to hold the asset in positive amounts. Their supply is the main force on the market in that stage of the cycle, which dominates the low positive demand of the fundamentalists and the positive price correction by the market maker.

The discussion will have made it clear that with position-based strategies, even though it is a prototype model and we have only studied the most regular periodic trajectories, the dynamic relationships are more involved than in the Beja-Goldman model; since in addition to the relationship between demand and price changes, the relationships between the two speculative groups of agents, and the new influence of the market maker's risk aversion, one also has to consider the relationships between the agents' stock and flow variables, i.e., their desired positions and the market orders deriving from them.

It can moreover be said that fundamentalists and chartists perform different roles over the cycle in the two modelling frameworks. Thus, take the stage of the cycle when after the peak the price begins to fall. In the BG model this phase is characterized by the negative demand of the fundamentalists, which dominates the positive demand of the chartists. By contrast, in the present PBT model chartist and fundamentalist demands have opposite signs from BG, and it is the negative demand of the chartists which dominates the positive demand of fundamentalists and so causes the price to fall.

The upper part of Figure 4 also keeps track of the agents' positions in the BG model, as they are implied by their demands and the corresponding trading activities. In the present case with the regular oscillations of demand and price, the positions, too, move in a periodic manner. The center of these oscillations, however, depends on the starting 
conditions. In the first three panels the initial values of $a^{m}, a^{f}, a^{c}$ have therefore been chosen in such a way that the positions oscillate around zero; see the thin solid (red) lines in Figure 4, which have been suitably rescaled to let the cyclical patterns and comovements stand out more clearly.

With the time series of the price and the positions we can compute the profits from the different trading strategies. To ease the discussion, let us assume that the agents' target positions are zero. Denoting the actual asset price by $P=\exp (p)$ and the value of the alternative asset that an agent $h$ holds by $m^{h}$, the wealth of this agent is given by $W^{h}=P a^{h}+m^{h}$. The changes in $m^{h}$ are inversely related to the sales and purchases of the risky asset, i.e., $\dot{m}^{h}=-P d^{h}=-P \dot{a}^{h}$. Hence the capital gains $g^{h}$ of agent $h$ from trading are determined by, ${ }^{8}$

$$
g^{h}=\dot{W}^{h}=\dot{P} a^{h}+P \dot{a}^{h}+\dot{m}^{h}=\dot{P} a^{h}=\exp (p) \dot{p} a^{h} \quad h=f, c, m
$$

These profits are depicted as the dotted (green) lines in Figure 4. Regarding the BG model, it is obvious that the fundamentalists should make positive profits over a cycle; after all they consistently buy low and sell high. Even their instantaneous profits turn out to be positive whenever the price changes, since they have gone long when the price is rising and short when it is falling.

Chartists, by contrast, earn positive profits only over short subintervals of the cycle: in the upswing (downswing) of the price just after their position becomes positive (negative). As soon as the price reaches its peak or trough, respectively, the chartists make losses again. It may also be observed that although the oscillations of the price and the position appear almost perfectly symmetrical, the induced motions of the profits are not. The losses of chartists are heavier when their long position is deteriorated by the falling price than when their position is negative and the asset appreciates.

Averaging over a full cycle, the losses of the chartists turn out to be almost equal to the profits earned by the fundamentalists. Therefore, in the present stylized scenario, the market maker neither loses nor wins from his function as an intermediary. The reason for this is that profits in the aggregate cancel out, $g^{f}+g^{c}+g^{m}=0$ in every instant of time, which follows from $(5)$.

\footnotetext{
${ }^{8}$ Recall that we neglect dividends, interest payments and similar proceeds from the assets. Zero transaction and information acquisition costs will likewise be understood.
} 
The second part of Figure 4 demonstrates that relative profits in the PBT model are different from the BG model. The PBT dynamics allows both fundamentalists and chartists to make positive profits over the cycle, which goes at the expense of the market maker. The latter could, of course, make up for these losses by charging a fee on the transactions or by bid-ask spreads, which so far have not been explicitly modelled.

Besides the qualitative statements, the profits of fundamentalists and chartists cannot be unambiguously compared, since the coefficients $\kappa_{f}, \kappa_{c}$, which for short have been referred to as "trading capital", incorporate the aggressiveness of single agents as well as the market fractions of the two groups. Hence if the almost ten times higher profits of the chartists that we obtain, $g^{c} / g^{f}=930 / 95$, were attributed to a ten times higher market fraction of this group, fundamentalist and chartist trading strategies would perform equally well. More informative in this respect is perhaps the ratio of the amplitudes of profits and positions, which are indeed almost equal: ${ }^{9} g^{f} / a^{f}=712 / 464 \approx 1.5$ versus $g^{c} / a^{c}=392 / 257 \approx 1.5$. Nevertheless, as long as both groups of speculative traders earn positive profits, unambiguous comparisons between the two can only be made across different parameter sets with identical coefficients $\kappa_{f}$ and $\kappa_{c}$.

\section{A stochastic version of the model}

\subsection{Properties of a base scenario}

Financial market models with a more or less distant view to the empirical stylized facts are directly set up as stochastic models. Often, but not always, a deterministic skeleton is then identified and part of the investigation is concerned with the deterministic properties and how they may influence the statistical properties of the full model. The presentation in this paper is the other way around. After having designed a deterministic prototype model and studied its main features, we now proceed with a minimal stochastic extension. This means the model is formulated in discrete time $(\Delta t=1$, so to speak) and we work with the widely employed assumption that the fundamental value follows a random walk.

\footnotetext{
${ }^{9}$ In view of the asymmetric peaks and troughs, the 'amplitude' is defined as the mean of the absolute values of the two.
} 
When establishing a numerical set of parameters that can serve as a base scenario and, in particular, in order to assess the memory $\theta$ of the chartists, the time unit of the model must be made explicit. To this end we make a visual inspection of several episodes of the S\&P 500 stock price index that are not too much trending, so that they allow us to recognize some (noisy) cyclical patterns. We perceive an average length between 7 and 8 days of these cycles, whatever may have caused them or how spurious the phenomenon might be. On the other hand, the period of the oscillations of (PBT) in Figure 4 was 55 time units, and the period in the model that will be relevant to us shortly below is 53 time units. Therefore, we may stipulate that seven time units of the model correspond to one trading day.

In many simulations of financial markets with a random walk for the fundamental value, the latter is supposed to change every day. To be in line with this convention, the fundamental value $v=v_{t}$ in PBT is updated, not every time unit, but every $\tau=7$ time units. Numerically we follow He and Li (2007, p. 3404), who adopt an annual volatility of $20 \%$. The corresponding standard deviation for the daily changes of $v_{t}$ is $\sigma_{v}=0.20 / \sqrt{250}=0.0126$. The precise formulation of this discrete-time stochastic process is given in Appendix A.3 .

Of course, since nonlinearities continue to be absent in the model, only parameters entailing deterministic stability are admissible to simulate this market. Our idea is to maintain the basic cyclical tendencies we have found, so that the parameters should give rise to dampened oscillations in the deterministic version (as in He and Li, 2007, pp. 3404f). Specifically, starting out from the PBT parameters in (16) above, we decide to settle down on a set of rounded parameters that imply a dampening factor of 0.69 and a period of 53 time units, i.e. 7.57 days (defining the dampening factor as the ratio of two successive peak values of the log price). These parameters are collected in Table 2 .

\begin{tabular}{ccccccc}
$\kappa_{f}$ & $\kappa_{c}$ & $\theta$ & $\lambda$ & $\mu$ & $\sigma_{v}$ & $\tau$ \\
\hline 0.20 & 0.57 & 20 & 1.00 & 0.050 & 0.0126 & 7
\end{tabular}

Table 2: Base scenario of the stochastic PBT model.

Figure 5 contrasts one year of daily data on the S\&P 500 index with 250 days = 1750 time units of a sample run of the base scenario. If one takes the limited noise in the model into account then, at face value, the empirical and artificial price series are fairly 
well comparable. For our investigations of the parameter effects below, this qualitative correspondence will be good enough.
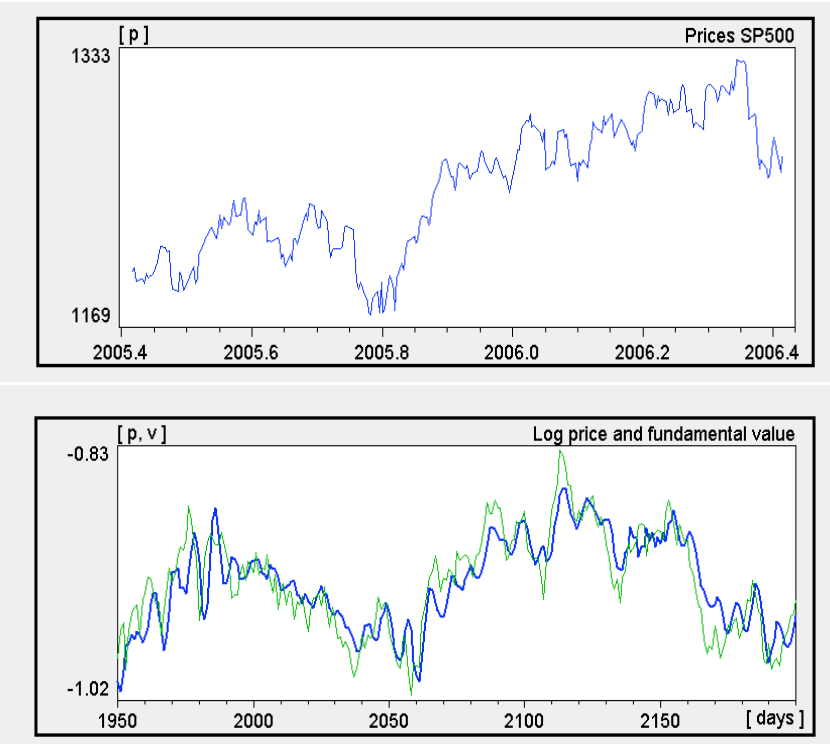

Figure 5: Contrasting an artificial price series with S\&P 500.

Note: Underlying the simulation run are the coefficients of Table 2. The bold (blue) line in the lower panel depicts the log price $p_{t}$, the thin (green) line is the random walk of the fundamental value $v_{t}$.

The lower panel in Figure 5 also illustrates that the price tracks the fundamental value rather closely (actually more closely than the examples given in Farmer, 2001, p. 67 and Farmer and Joshi, 2002, pp.162f). Upon closer inspection of the price series over shorter time intervals, however, one could distinguish episodes where $p_{t}$ seeks to catch up with $v_{t}$ very rapidly, and other episodes of 10 or 20 days where the temporarily divergent tendencies of the cycle generating mechanism prove to be dominant, so that $p_{t}$ and $v_{t}$ move out of line.

The fact that the price does not deviate very far and not very persistently from the fundamental value implies that the positions $a_{t}^{f}$ of the fundamentalists center around their target. Though it is analytically not obvious, it turns out that the basic convergence forces in the system are so strong that also the positions $a_{t}^{c}$ of the chartists are centered. This notwithstanding, their amplitude can be quite variable over longer time intervals.

The short sample period of 50 days shown in Figure 6 can illustrate three things. First, the consistent centering of the agents' positions. Second, the distinctly larger fluctuations of $a_{t}^{c}$ versus $a_{t}^{f}$ (note the equal scaling of the axes). Hence the market maker's inventory $a_{t}^{m}$ 

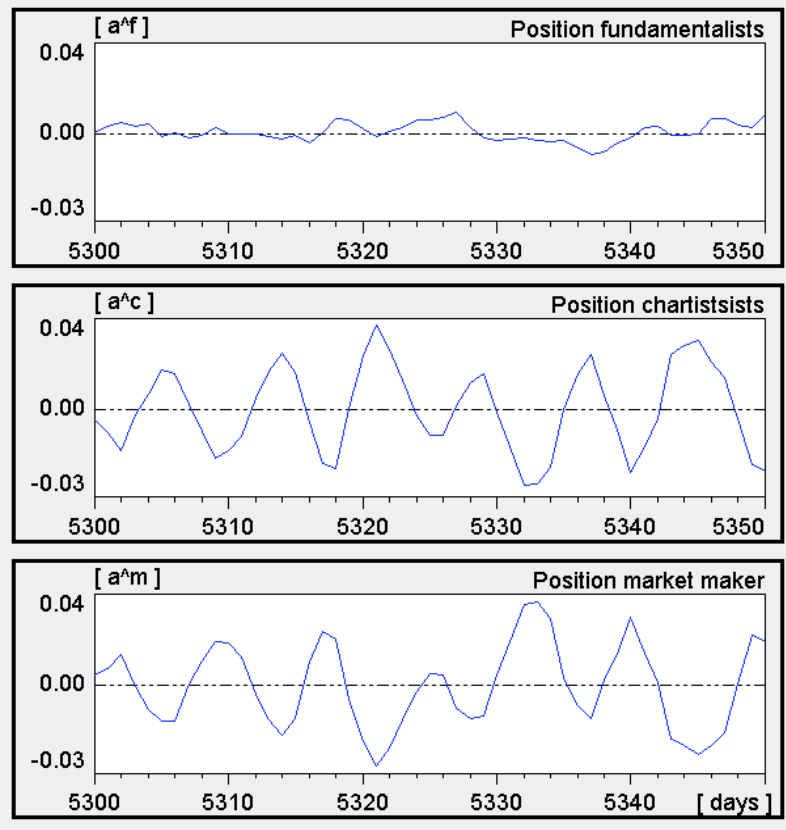

Figure 6: Positions $a_{t}^{f}, a_{t}^{c}, a_{t}^{m}$ over a short sample period in the base scenario.

is largely inversely related to $a_{t}^{c}$, which demonstrates that his main function is to buffer the market disequilibria caused by the chartists. A third point is that the cyclical tendencies of the deterministic core manifest themselves, not so much in the price series with its pseudo trend incidents, but at the level of the positions of the chartists and the market maker.

Regarding the capital gains that the agents derive from their positions, the regular oscillations in Section 3.4 proved to yield positive profits over the cycle for fundamentalists as well as for chartists, which goes at the expense of the market maker. Figure 7 makes it evident that, in the long-run, this also holds true in the stochastic setting, at least at the present parameter values. Precisely, the diagram presents the daily profits of agent group $h, g_{t}^{h}=W_{t}^{h}-W_{t-\tau}^{h}$ with $\tau=7$ (period $t$ a multiple of $\tau$ ) and $h=f, c, m$.

The high spikes in the middle panel for the chartists might suggest that the profits from $a_{t}^{c}$ are much higher than the profits from $a_{t}^{f}$, similar perhaps to the ratio 930/95 reported for the deterministic motion. These appearances are, however, deceiving. Over the two years $=500$ days shown in Figure 7 , the average daily profits of fundamentalists and chartists are 1.26 and 2.87, respectively (both multiplied by $10^{5}$ ). Over the long run of 100 years (i.e. 25,000 days or 175,000 time units), these magnitudes are 0.95 and 2.17, respectively. Even if one were to assume an identical number of agents in both groups, it 

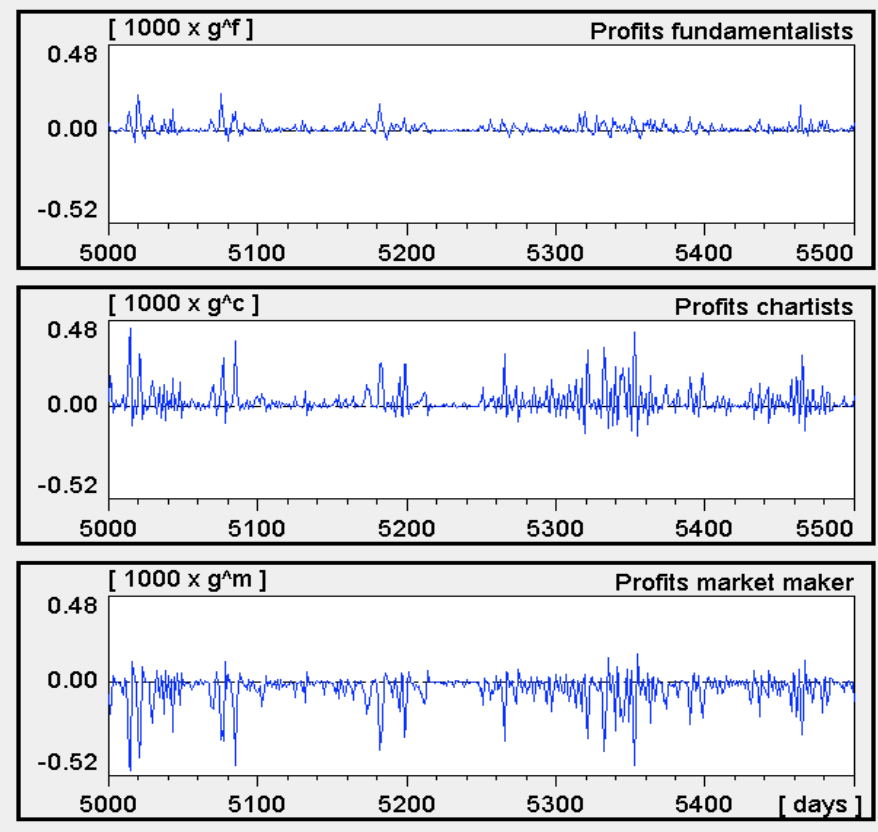

Figure 7: Daily profits $g_{t}^{f}, g_{t}^{c}, g_{t}^{m}$ of the agents in the base scenario.

could be argued that fundamentalism and chartism are about equally attractive, since the higher profits $g_{t}^{c}$ come along with a higher standard deviation, 6.29 versus 2.71 for $g_{t}^{f}$ (and 7.51 versus 2.88 in Figure 7 ). Note that in both cases the positive mean values are not much more than one third of the standard deviation. So chartist and also fundamentalist trading is not without risk — at least if the initial wealth is small or trading is debt financed.

It will also be observed that for the chartists tranquil times alternate with larger fluctuations of the profits. This phenomenon may be informally described as volatility clustering. Nevertheless, it must then not be forgotten that we are concerned with profits, and here the profits of only one of the speculative groups. Volatility clustering of returns, to which this mode of expression usually applies, is not yet achieved; in this respect the model is still too simple.

\subsection{A stochastic equilibrium notion}

To put the properties of one or several simulation runs into perspective, it would be expedient if one could refer to a notion of stochastic equilibrium. An obvious approach to meet this desire is to view the stochastic process as a Markov process, which is set up by 
the transition probabilities $P\left(d_{t}, B\right)$ that a price deviation $d_{t}:=p_{t}-v_{t}$ in period $t$ will, in the next period $t+1$, be contained in the set $B$. The period- $t$ variable to be studied will then be a time-varying unconditional probability distribution $\pi_{t}$ of $d_{t}$. If its law of motion is succinctly summarized by a mapping $\Phi$, such that $\pi_{t+1}=\Phi\left(\pi_{t}\right)$, an equilibrium of this process is constituted by an invariant distribution $\pi^{\star}$ satisfying the equation $\pi^{\star}=\Phi\left(\pi^{\star}\right)$. Under certain regularity conditions, which one feels should be satisfied here, such a fixed point exists, is unique, and is attractive, i.e., $\pi_{t} \rightarrow \pi^{\star}$ as $t \rightarrow \infty$ for any initial distribution $\pi_{0} \cdot{ }^{10}$

If this kind of ergodicity prevails, the qualitative long-run behaviour of each sample path of the system is completely described by the Markov equilibrium from a statistical point of view. It does not, however, describe the quantitative behaviour of single sample paths nor the qualitative behaviour of ensembles of sample paths, whether sample paths starting at different states converge or diverge for a given sequence of random shocks. An appropriate concept to treat this problem is that of a random fixed point and its asymptotic stability, which are counterparts of the deterministic concepts. It is, in particular, convenient that this notion of stability is one of pointwise convergence.

In its precise definition, a random fixed point is a random variable, that is, a mapping on a probability space, which is a rather abstract and technical concept. ${ }^{11}$ For the present purpose it is sufficient to degenerate the probability space to a given sequence $\left\{\delta_{t}\right\}_{t=0}^{\infty}$ of the random walk innovations to the fundamental value. In this perspective, the stochastic model becomes a forced oscillator, which has a deterministic solution path once an initial price history is given. An implication of a random fixed point, then, is that the solutions from any two price histories get, and stay, arbitrarily close as time unfolds. As a consequence, all these sample paths have the same limiting behaviour. ${ }^{12}$

\footnotetext{
${ }^{10}$ Such a result should at least be obtainable if the normal distribution for the innovations in the random walk is truncated. For a compact introduction to the these discrete-time Markov processes with continuous state space, and the conditions for uniqueness of and convergence toward $\pi^{\star}$, see Futia (1982),

${ }^{11}$ Arnold (1998) is a standard reference to the theory of random dynamical systems. For an immediate introduction to random fixed points and applications to economic systems, see Schenk-Hoppé and Schmalfuß (2001), and Böhm and Chiarella (2005).

${ }^{12}$ The statistical properties of the associated invariant distribution could thus be determined from the limits of orbits, since by ergodicity any empirical time average (or other moment) converges to the true mean (or other moment) of the invariant distribution (Böhm and Chiarella, 2005, p. 77).
} 

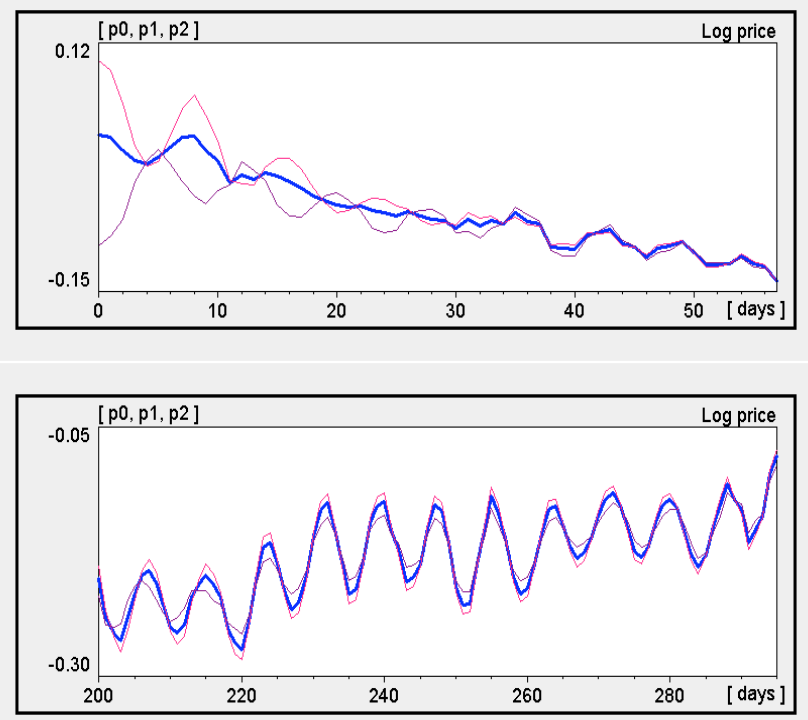

Figure 8: Convergence of sample paths from different initial conditions.

Note: The upper panel has the base scenario underlying with, in particular, $\kappa_{c}=0.57$. In the lower panel this parameter is changed to $\kappa_{c}=0.61$, which is close to the bifurcation value.

Figure 8 illustrates the convergence process to the limiting behaviour for the base scenario of Table 2. Actually, convergence is rather fast, after 40 days the three price series are virtually identical. A lower speed of convergence will, however, be expected if the parameters are closer to the stability frontier. This intuition is confirmed in the lower part of Figure 8, where with $\kappa_{c}=0.61$ the chartist trading capital is only marginally below its bifurcation value. Given the large amplitude of the price fluctuations around the (omitted) path of the fundamental value (cf. the scale in the two panels), it is remarkable that these cycles synchronize already much earlier. It follows that generally, if one is interested in certain summary statistics as being representative of a given parameter set, there will be no problems with the sampling variability from different starting conditions. Already a few explorations of the kind here presented give a good feeling for the length of an initial transition period that should be ignored. For the statistics computed in the experiments below, the first 500 days will be discarded, with which we are completely on the safe side. 


\subsection{Parameter effects}

In this section we return to the parameters of the model and an analysis of their properties. Our first aim is to characterize the parameters as stabilizing or destabilizing, which requires a new effort since the notion of stability in a stochastic setting is different from deterministic stability. There we had a qualitative distinction, according to which the equilibrium is either attractive or not. Variations in something like a degree of stability could only be informally considered by alluding to the distance of a parameter from the stability frontier; the implicit idea that such a distance may be inversely related to a speed of convergence toward the equilibrium has not been pursued.

In the present context, the concept of 'stability' can be quantified in a very natural way. As the basic convergence of the deterministic skeleton is continuously perturbed by the random shocks to the fundamental value, which again and again move this target away from the current price, the relative strength of the centripetal and centrifugal forces is most directly measured by the average gap between price and fundamental value. In other words, the central figure with which we will be concerned is the misalignment, specified as the long-run standard deviation of $p_{t}-v_{t}$ and denoted as $\sigma(p-v)$. The time series in Figure 5 was already sufficiently representative to show that the misalignment is quite limited in our base scenario, and we will now ask how it is influenced by ceteris paribus variations of the single parameters. As indicated above, the 'long-run' perspective for this and all other summary statistics will be defined by a period of 25,000 days. ${ }^{13}$

If we recall the models on position-based trading in the literature and their concern with the cointegration of prices and values, then the parameter of primary interest to us is the risk aversion of the market maker, $\mu$, which has been said to be in control of this feature. It has been observed in that literature that prices and values disconnect if there are only a few homogeneous groups of agents and, in our notation, $\mu$ is zero. Surely, the phenomenon should essentially persist if $\mu$ is only slightly raised. On the other hand, from the comment on Proposition 5 we know that high values of $\mu$ will eventually destabilize the deterministic equilibrium. Hence the misalignment should also become increasingly worse as $\mu$ approaches its bifurcation value $\mu^{H}$ from below (the letter $H$ because it is a Hopf bifurcation).

\footnotetext{
${ }^{13}$ After dropping the first 500 days to eliminate any impact of the starting values on the simulations.
} 
This argument can be readily tested with the parameters from Table 2 , where $\mu$ varies between zero and $\mu^{H}$ and the latter is computed as $\mu^{H}=0.0856$. The sequence of the random shocks to the fundamental value is the same in each simulation run (this equally holds for the other experiments to follow). The top-left panel of Figure 9 shows the resulting misalignment over this range as a function of $\mu$, which indeed displays the predicted Ushape. Hence, regarding the present notion of stability in the stochastic framework, the market maker's risk aversion is an ambiguous parameter.

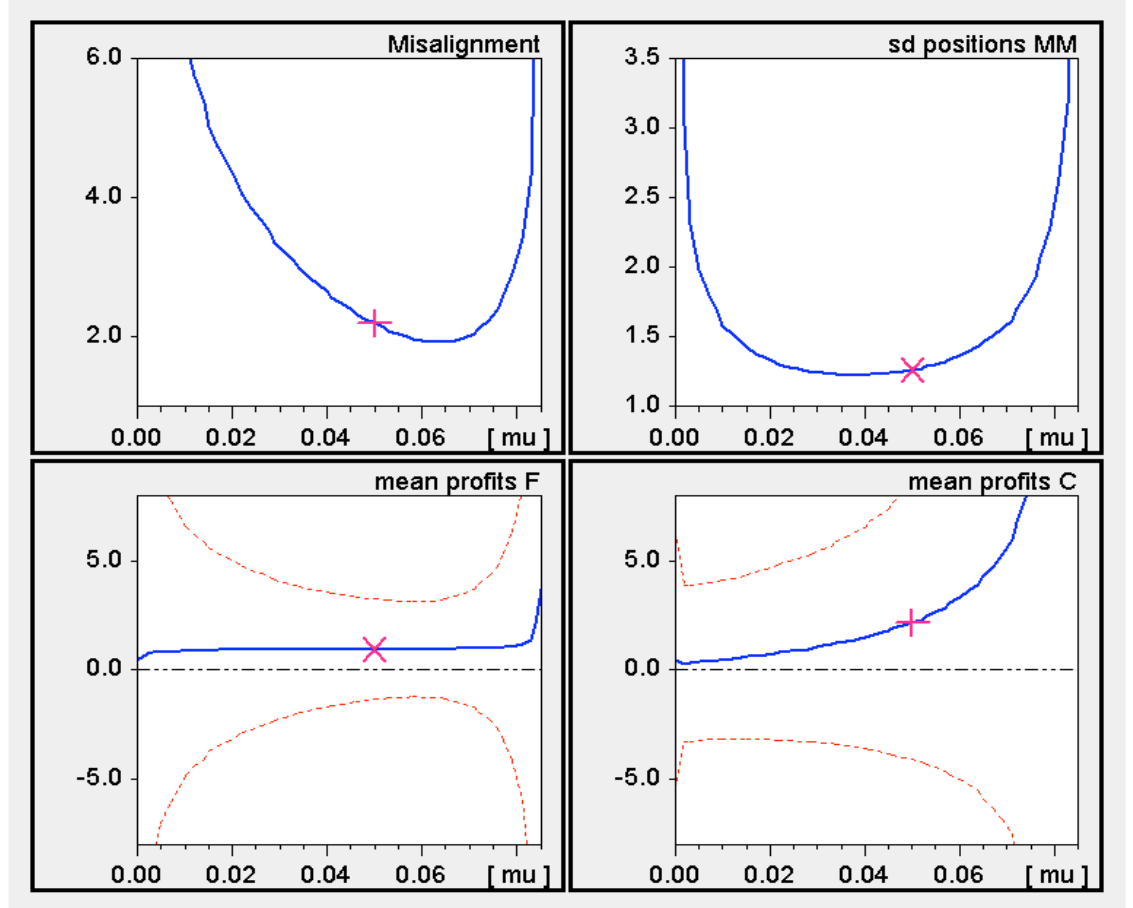

Figure 9: Variations of $\mu$ in the base scenario.

Note: Based on samples of 25,000 days. Misalignment is specified as $\sigma(p-v)$ over this period (in percent), 'sd positions MM' is $\sigma\left(a^{m}\right)$. The dotted lines indicate a one standard deviation band around the mean profits. The crosses mark the base scenario.

In an immediate thought experiment it can here be asked why the market maker should choose a value of $\mu$ that entails a relatively weak misalignment (especially if he has no real-time knowledge of the fundamental value). Since his main motive is to limit the deviations of his inventory from target, we additionally compute the long-run standard deviation $\sigma=\sigma\left(a^{m}\right)$ of his positions, the outcome of which is plotted in the top-right panel of Figure 9. While it will not be surprising that a strong (weak) misalignment goes along 
with strong (weak) imbalances, it is remarkable that the 'valley' in the graph of the function $\mu \mapsto \sigma\left(a^{m}\right)$ is considerably wider than in the misalignment function $\mu \mapsto \sigma(p-v)$.

Having above computed the profits that the speculative agents earn with their strategies, we can furthermore examine how seriously they are affected by different degrees of the market maker's risk aversion. The corresponding mean values over the 25,000 days, $\bar{g}^{f}$ and $\bar{g}^{c}$, are drawn in the two bottom panels of Figure 9. Two features are here worth mentioning. First, it cannot be generally concluded that a strong misalignment is favourable or unfavourable for fundamentalists; this rather depends on the mechanism causing the misalignment. If the prices disconnect from the fundamental in one direction over longer periods of time, which will be the typical case at low values of $\mu$, then there are only weak prospects for the position-based fundamentalists to extract profits from that pattern. Better opportunities, however, arise for them if the misalignment is caused by wider and wider fluctuations of the price around the fundamental value, as they occur when the risk aversion approaches the bifurcation value $\mu^{H}$.

The second feature is the temporal structure, which becomes more pronounced as $\mu$ rises and so provides technical traders with more or stronger regularities in the price series that they can seek to exploit. The lower-right panel of Figure 9 cogently confirms this intuition. The profits of the chartists in this model are seen to be increasing with $\mu$, even at an increasing rate and without any further adjustments in the trading coefficient. It should, however, be observed that the associated risk rises, too. More specifically, the standard deviation of the profits tend to become unbounded as $\mu$ gets closer to $\mu^{H}$.

Nevertheless, all of these findings should not be prematurely generalized. To illustrate this, Figure 10 presents the results from the same experiments as before; this time for a market which, owing to a high trading capital $\kappa_{f}$ of the fundamentalists, is not only stable for all values of $\mu$, but where convergence in the deterministic version is also monotonic. ${ }^{14}$ In this example, even much larger values of $\mu$ than above are not able to generate sufficient temporal structure in the price from which the chartists could profit to an appreciable extent; at least not in comparison to the group of fundamentalists, for which this kind of dynamics is more favourable.

\footnotetext{
${ }^{14}$ Evidently, $\kappa_{c}<\kappa_{f} / 2$ for the parameters in the note on Figure 10, so that the condition in Proposition $3(a)$ is satisfied.
} 


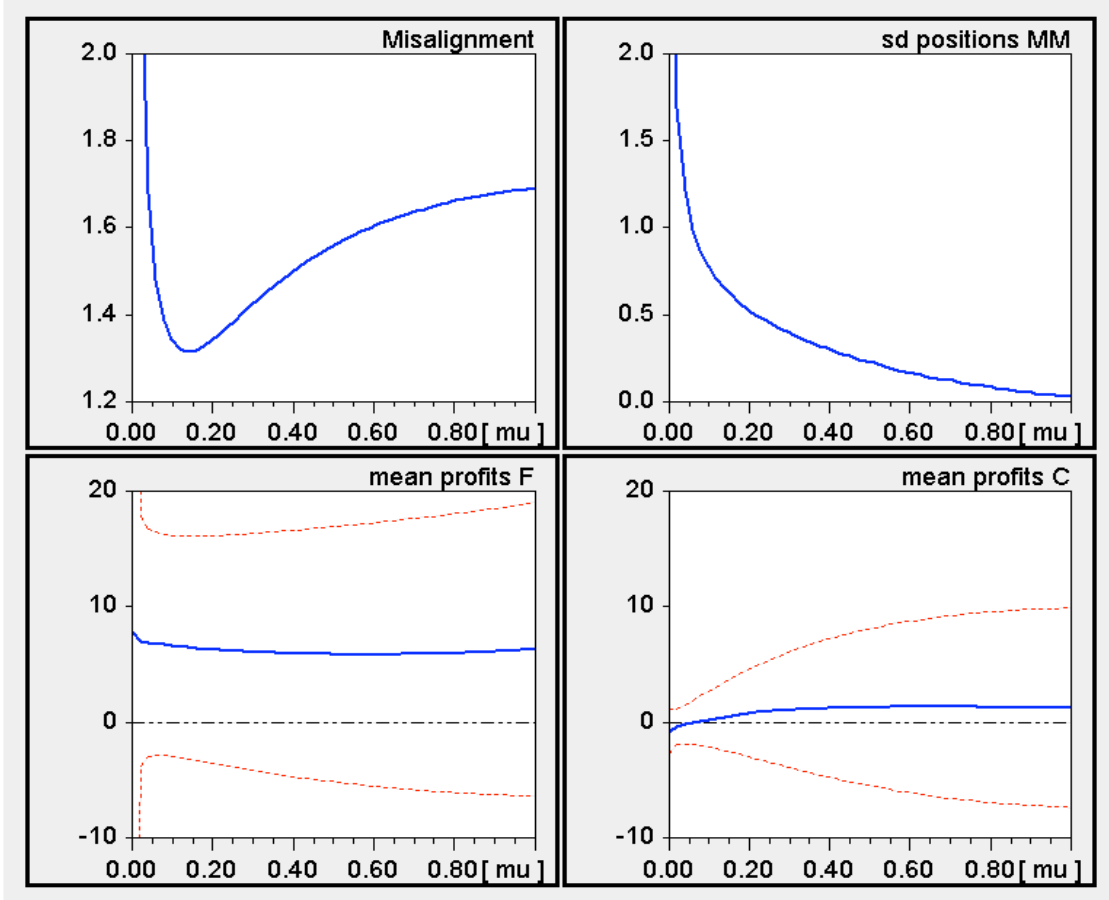

Figure 10: Variations of $\mu$ in a stable scenario with monotonic convergence.

Note: Maintaining $\lambda, \theta, \sigma_{v}$ and $\tau$, the two trading capital coefficients are $\kappa_{f}=0.80$ and $\kappa_{c}=0.25$.

As regards the risk of the market maker, he can drive the standard deviation of his inventory down to zero by sufficiently increasing his price correction coefficient $\mu$; cf. the top-right panel in Figure 10. The reduced fluctuations in $a^{m}$ are, however, not tantamount to a similar decrease in the misalignment of the price. After the initial decline of $\sigma(p-v)$ as $\mu$ rises above zero, this function begins to increase again as $\mu$ rises further, until it eventually levels off at $1.70 \%$. The top-left panel is thus another instance which shows that the effects of the market maker's risk aversion are not easily assessed.

We believe that the cyclical base scenario is a more attractive and fruitful numerical specification than a scenario of overall monotonic convergence, and we will therefore return to it. The experiments in Figure 10 are, however, useful for a better evaluation of the previous results and the ones to follow, insofar as they point out that cyclicality in the deterministic skeleton may be of crucial importance for them to come about.

Concentrating on the numerical coefficients from Table 2, we can take the other parameters $\kappa_{f}, \kappa_{c}, \theta$ and $\lambda$, one after another, let them vary up or down to their bifurcation 
value, compute the same summary statistics as we did for $\mu$, and plot them as functions of this parameter. The corresponding diagrams, which are analogous to Figure 9, are documented in Appendix A.4. Here we report the main parameter effects, which are collected in Table $3 .{ }^{15}$

\begin{tabular}{lccccc}
\hline & weight F & weight C & memory C & liquidity & risk aversion MM \\
& $\kappa_{f}$ & $\kappa_{c}$ & $\theta$ & $\lambda$ & $\mu$ \\
\hline Misalignment : & $\mathrm{S}$ & $\mathrm{A}$ & $\mathrm{A}$ & $\mathrm{A}$ & $\mathrm{A}$ \\
Profits F : & $\mathrm{H}$ & $(\mathrm{H})$ & $(\mathrm{L})$ & $(\mathrm{L})$ & $(\mathrm{H})$ \\
Profits C : & $\mathrm{L}$ & $\mathrm{H}$ & $\mathrm{L}$ & $\mathrm{L}$ & $\mathrm{H}$ \\
\hline
\end{tabular}

Table 3: Effects of ceteris paribus variations of the parameters in the (cyclical) base scenario.

Note: $\mathrm{S}$ and A indicate that the parameter is stabilizing or ambiguous, respectively, insofar as an increase of it reduces the misalignment, or produces both increases and reductions over different intervals. $\mathrm{H}(\mathrm{H})$ and $\mathrm{L}(\mathrm{L})$ means that an increase of the parameter leads to (weakly) higher or lower mean profits for the fundamentalists $(\mathrm{F})$ and chartists (C). Besides Figure 9, the diagrams from which this summary derives are given in Appendix A.4.

If we view a decrease (increase) of the misalignment brought about by a higher parameter value as a stabilizing (destabilizing) effect, then the first row in Table 3 can be compared to the stability effects in Table 1. It is thus seen that an increase in the trading capital of the fundamentalists is stabilizing according to both the stochastic and deterministic criterion. However, besides $\mu$ also the other three parameters $\kappa_{c}, \theta$ and $\lambda$ have an ambiguous impact on the misalignment. If, in finer detail, we consider 80 percent or more of the entire parameter interval, over which $\sigma(p-v)$ happens to change in one direction, then it could be said that $\theta$ and $\lambda$ tend to be weakly destabilizing, and $\kappa_{c}$ tends to be weakly stabilizing. This, however, is just the opposite characterization from Table 1.

The last two rows of Table 3 summarize the impact of the parameter variations on the long-run profits of fundamentalists and chartists. Clear effects are produced by the

\footnotetext{
${ }^{15}$ Concerning the variations of $\theta$, we use specification (13) for the chartist positions. $\tilde{\kappa}_{c}$ is fixed at the value it attains in the base scenario, where it satisfies the equation $0.57=\kappa_{c}=\tilde{\kappa}_{c} / \theta=\tilde{\kappa}_{c} / 20$. The appendix, in particular, shows that the shapes of the functions of the statistics are very similar for the variations of $\lambda$ and $\theta$.
} 
fundamentalist trading capital. A ceteris paribus increase of $\kappa_{f}$ grants the fundamentalists higher mean profits and reduces those of the chartists. By contrast, the changes of the fundamentalist profits brought about by the other parameters are weak or even negligible, except perhaps (very) close to the stability frontier. The effects are in any case more distinct for the capital gains of the chartists. They profit from a rise in $\mu$ (already noted) and their own trading capital $\kappa_{c}$, from a shorter memory $\theta$, and from less liquidity $\lambda$ on the market.

On the whole, Table 1 in Section 3.2 above is the upshot of the stability analysis of the deterministic system, and Table 3 compactly sums up the central dynamic properties of the stochastic PBT model.

\section{Conclusion}

Within the field of asset pricing models with few groups of heterogeneous traders, we have started out from the observation that the overwhelming majority of these models leaves the positions of the agents as a residual in the background. As a consequence, the positions may easily become unbounded once some stochastic perturbations are introduced. This inconsistency can be avoided by modelling the agents' strategies as position-based rather than order-based. There are a few examples of this approach in the recent literature, but it seems they have made relatively little impact as yet. One reason for the limited influence might be the lack of an elementary reference model with position-based trading. The main purpose of the present paper was therefore the design and analysis of such a prototype model, whose properties are well understood and which, in particular, can be contrasted with an order-based prototype model.

More specifically, we extracted a position-based prototype model from two major contributions by Farmer (2001) and Farmer and Joshi (2002), while as a order-based reference model the Beja-Goldman model was most suitable. A special feature of our model, which has no counterpart in the latter, is that it allows us to discuss the role of the market maker at a similarly elementary level as the other components of the two models. Under certain conditions, his risk aversion introduces more temporal structure into the price dynamics and potentially even destabilizes it.

Although the deterministic continuous-time version of the model is mathematically (much) more involved than the Beja-Goldman model, the stability conditions on the para- 
meters turned out to be completely analogous. Hence the present framework should provide an equally fruitful and promising basis for conceptually more ambitious extensions, which may be concerned with time-varying demand intensities of fundamentalists and chartists, or with switching market fractions of the two, or more, speculative groups. ${ }^{16}$ In this respect we hope that the results from our numerical study of the stochastic market can give some useful insights, regarding the misalignment of price and fundamental value as well as the differential profits of fundamentalists and chartists. ${ }^{17}$ It is a challenging problem whether models developed in this direction will be able to match (some of) the stylized facts of volatility clustering, fat tails and power-law behaviour. In any case, these models will not be plagued with inconsistencies regarding the long-run evolution of the agents' positions.

\section{A Appendix}

\section{A.1 Proof of Proposition 3}

Proof: To prove part (a) of Proposition 3, write system (PBT) as

$$
\dot{p}(t)=a p(t)+b p(t-\theta)+c \dot{p}(t-\theta)
$$

This notation allows us to refer directly to a simplified version of Theorem 1 and Corollary 1 in $\mathrm{Hu}$ and $\mathrm{Hu}(1996) .{ }^{18}$ It says that (18) is asymptotically stable if $|c|<1$ and if for all complex numbers $\xi=x+i y$ on the border of the unit circle, where $|\xi|=x^{2}+y^{2}=1$, the real part of $(1-\xi c)^{-1}(a+\xi b)$ is negative.

With the formula $(\alpha+i \beta)^{-1}=(a-i \beta) /\left(\alpha^{2}+\beta^{2}\right)$ for the inverse of complex numbers, we have $\operatorname{Re}\left[(1-\xi c)^{-1}(a+\xi b)\right]=\operatorname{Re}[(1-c x-i c y)(a+b x+i b y)]=\operatorname{Re}[(1-c x+i c y)(a+b x+$ $\left.i b y)] /\left[(1-c x)^{2}+c^{2} y^{2}\right]\right)$. Substituting $1-x^{2}$ for $y^{2}$, the sign of this expression is easily seen to be determined by $f(x):=a-b c-(a c-b) x$, where $x$ varies between -1 and 1 . Since $f(\cdot)$ is

\footnotetext{
${ }^{16}$ An elaborate attempt with switching market fractions is Pape (2007).

${ }^{17}$ Given the good performance of chartists in scenarios with some cyclical potential, and the dominance of fundamentalist profits in the Beja-Goldman model and other models with order-based strategies, it appears a fascinating task to pair our present position-based chartists with the order-based fundamentalists from Beja and Goldman to see what kind of market ecology may emerge from that. The fundamentalists should perhaps be endowed with some correction mechanism to keep their positions within bounds.

${ }^{18}$ In its generality, their theorem applies to vectors $p=p(t)$ and square matrices $A, B, C$ in place of the real numbers $a, b, c$.
} 
linear, only the end-points have to be considered, for which we obtain $f(-1)=(a-b)(1+c)$ and $f(1)=(a+b)(1-c)$.

The sign of $(a+b)$ and $(a-b)$ is given by $-\mu\left(\kappa_{f}-\kappa_{c}\right)-\mu \kappa_{c}=-\mu \kappa_{f}$ and $-\mu\left(\kappa_{f}-\right.$ $\left.\kappa_{c}\right)+\mu \kappa_{c}$, respectively. The latter expression is negative if $\kappa_{c}<\kappa_{f} / 2$. $(1-c)$ and $\left.1+c\right)$ are positive if $|c|=\kappa_{c} /\left(\lambda-\kappa_{c}+\kappa_{f}\right)<1$, which is equivalent to $\kappa_{c}<\kappa_{f} / 2+\lambda / 2$. Hence $\kappa_{c}<\kappa_{f} / 2$ is a sufficient condition for $f(-1)<0$ as well as $f(1)<0$, and it can be concluded that the conditions stated in the abovementioned theorem are fulfilled.

The argument for proving part (b) is borrowed from Barker and Whitesides (1976, pp. 759ff). For better reference to this article we now write (PBT) in the form

$$
a_{0} p(t)+a_{1} \dot{p}(t)+b_{0} p(t-\theta)+b_{1} \dot{p}(t-\theta)=0
$$

from where the characteristic equation for the roots $s \in \mathbb{C}$ reads

$$
Q(s):=a_{0}+b_{0} \exp (-\theta s)+\left[a_{1}+b_{1} \exp (-\theta s)\right] s=0
$$

and the coefficients in (19) are given by the structural parameters as

$$
\begin{array}{ll}
a_{0}=\mu\left(\kappa_{f}-\kappa_{c}\right) /\left(\lambda+\kappa_{f}-\kappa_{c}\right) & a_{1}=1 \\
b_{0}=\mu \kappa_{c} /\left(\lambda+\kappa_{f}-\kappa_{c}\right) & b_{1}=\kappa_{c} /\left(\lambda+\kappa_{f}-\kappa_{c}\right)
\end{array}
$$

It has to be shown that $Q(\cdot)$ has a root with positive real part, since this implies the instability of (19). Given that all roots $s=\sigma+i \nu$ must satisfy the inequality

$$
|| a_{1}|-| b_{1}|\exp (-\theta \sigma)||s| \leq\left|a_{0}\right|+\left|b_{0}\right| \exp (-\theta \sigma)
$$

and $a_{1}$ and $b_{1}$ are different from zero, the roots of $Q(\cdot)$ have bounded real part $\sigma$. Arranging the roots in ascending order of their modulus, it can more specifically be concluded that the real parts of the large modulus roots $s_{k}$, for which $\left|s_{k}\right| \rightarrow \infty$ as $k \rightarrow \infty$, obey the constraint

$$
\lim _{k \rightarrow \infty}\left[\left|a_{1}\right|-\left|b_{1}\right| \exp \left(-\theta \sigma_{k}\right)\right]=0
$$

Hence their real parts get arbitrarily close to $\sigma_{\infty}=\sigma_{\infty}(\theta):=-(1 / \theta) \ln \left(\left|a_{1} / b_{1}\right|\right)$. It remains to observe that under the restriction $\kappa_{c}<\lambda+\kappa_{f}$, the inequality $\kappa_{c}>\left(\lambda+\kappa_{f}\right) / 2$ is equivalent to $\left|a_{1} / b_{1}\right|=\left(\lambda-\kappa_{c}+\kappa_{f}\right) / \kappa_{c}<1$ : since $\sigma_{\infty}>0$ in this case, there is one root $s_{k}$ (indeed infinitely many) with positive real part $\sigma_{k}$ 
Regarding the third part of the proposition, we first observe that $\kappa_{c}>\kappa_{f} / 2$ is tantamount to $\kappa_{c}>\kappa_{f}-\kappa_{c}$, so that the numerator in the definition of $\nu^{\star}$ is positive. The other condition $\kappa_{c}<\left(\lambda+\kappa_{f}\right) / 2$ gives $\lambda+\kappa_{f}-\kappa_{c}>\kappa_{c}$, so that the denominator is positive as well.

The next step follows the reasoning in Barker and Whitesides (1976, p. 761). Again consider the characteristic polynomial $Q(\cdot)$ as it is given in $(20)$. For $\theta \rightarrow 0(\theta>0)$, it has one (real) root that is arbitrarily close to the one root of the polynomial equation $Q_{o}(s):=a_{0}+b_{0}+\left(a_{1}+b_{1}\right) s$, which is obviously negative. The remaining roots of $Q(\cdot)$ are complex and have arbitrarily large moduli. Let $\hat{\theta}$ be a number such that this situation prevails for all $\theta \leq \hat{\theta}$ and $\hat{\sigma}:=(1 / \hat{\theta}) \ln \left(\left|a_{1} / b_{1}\right|\right)$ is less than the root of $Q_{o}(\cdot)$; negativity of $\ln \left(\left|a_{1} / b_{1}\right|\right)$ derives from from $\kappa_{c}<\left(\lambda+\kappa_{f}\right) / 2$ and reversing signs in the argument following eq. (22). Since for the large modulus roots, $\sigma_{\infty}=-(1 / \theta) \ln \left(\left|a_{1} / b_{1}\right|\right) \rightarrow-\infty<\hat{\sigma}<0$ as $\theta$ approaches zero, $Q_{o}$ and $Q$ have the same number of roots whose real part exceeds the negative number $\hat{\sigma}$. This proves that system (PBT) is asymptotically stable if $\theta$ is sufficiently small.

The rest of the proof draws on Ford and Nelson (2004). It focusses on any critical values $\theta^{\star}$ of the lag $\theta$ at which a root of the characteristic equation transitions from having negative real parts to having positive real parts. When this occurs, the root must be purely imaginary; $s=0$ in (20) is not possible by virtue of $a_{0}+b_{0}>0$, which establishes the Hopf bifurcation statement of part $(c)$. The main point in the proof of part $(d)$ is that this root $s=i \nu=i \nu^{\star}$ can be computed independently of the lag $\theta$. In fact, the critical value $\theta^{\star}$ that gives rise to just this root $i \nu^{\star}$ is subsequently determined from one of the real and imaginary components of the characteristic equation.

To compute the root $s=i \nu^{\star}$, we begin by separating the characteristic polynomial (20) into its real and imaginary parts. Writing the exponential $\exp (-i \nu)$ in terms of the trigonometric functions, it reads

$$
Q(i \nu)=a_{o}+i a_{1} \nu+\left(b_{0}+i b_{1} \nu\right)[\cos (\nu \theta)-i \sin (\nu \theta)]=0
$$

In order for (20) to hold, both the real and imaginary part must be 0 , which yields the two real-valued equations

$$
\begin{array}{r}
a_{0}=-\left[b_{0} \cos (\nu \theta)+b_{1} \nu \sin (\nu \theta)\right]=: \quad f_{1}(\nu, \nu \theta) \\
a_{1}=b_{0} \sin (\nu \theta)-b_{1} \nu \cos (\nu \theta)=: \quad f_{2}(\nu, \nu \theta)
\end{array}
$$


The trigonometric terms disappear and the lag $\theta$ is eliminated if the two equations are squared and then added up. In this way the condition $a_{0}^{2}+a_{1}^{2} \nu^{2}=b_{0}^{2}+b_{1}^{2} \nu^{2}$ is obtained. It is solved for $\nu=\nu^{\star}$ as

$$
\nu^{\star}=\sqrt{\frac{b_{0}^{2}-a_{0}^{2}}{a_{1}^{2}-b_{1}^{2}}}=\sqrt{\frac{\mu^{2}\left[\kappa_{c}^{2}-\left(\kappa_{f}-\kappa_{c}\right)^{2}\right]}{\left(\lambda+\kappa_{f}-\kappa_{c}\right)^{2}-\kappa_{c}^{2}}}
$$

The second expression, which is the same as the one in the proposition, follows from definition (21) for the coefficients. For better reference in the further course of the proof, we also note

$$
b_{0}>a_{0} \quad \text { and } \quad a_{1}>b_{1}
$$

It will be established in a moment that there exist delay parameters $\tilde{\theta}>0$ such that the first equation in $(24)$ is satisfied, $f_{1}\left(\nu^{\star}, \nu^{\star} \tilde{\theta}\right)=a_{0}$. The critical values satisfy the second equation, too, since from the determination of $\nu^{\star}$ by squaring and adding the two equations one has $a_{1}^{2} \nu^{2}-f_{2}^{2}\left(\nu^{\star}, \nu^{\star} \tilde{\theta}\right)=f_{1}^{2}\left(\nu^{\star}, \nu^{\star} \tilde{\theta}\right)-a_{0}^{2}=0$. Hence changing the perspective and considering $\tilde{\theta}$ as given, the purely imaginary number $s=i \nu^{\star}$ is a root satisfying (24) and thus the characteristic equation (23) or (20).

The minimum value $\theta=\theta^{\star}$ that in eq. (24) equates $f_{1}\left(\nu^{\star}, \nu^{\star} \theta\right)$ to $a_{0}$ is contained in the interval $\left(0, \pi / \nu^{\star}\right)$. This is inferred from $f_{1}\left(\nu^{\star}, 0\right)=-b_{0}<a_{0}<b_{0}=f_{1}\left(\nu^{\star}, \pi\right)$, where $-b_{0}<a_{0}$ is obvious and the other inequality was already stated in (26). ${ }^{19}$ Clearly, if $\theta=\theta^{\star}$ for the lag parameter, the period of the system's cyclical motions is given by $T=2 \pi / \nu^{\star}$, and $T>2 \theta^{\star}$ follows from $\theta^{\star}<\pi / \nu^{\star}$.

Having seen that any root $s=s(\theta)$ of the characteristic equation crossing the imaginary axis is determined as $s= \pm i \nu^{\star}$, we now show that with rising $\theta$ the root must in any case move from the negative into the positive half-plane. This kind of transition comes about at all $\tilde{\theta}$ that, by equating $f_{1}\left(\nu^{\star}, \nu^{\star} \theta\right)$ to $a_{0}$, give rise to just this root. Formally, ${ }^{20}$

$$
\left.\frac{d}{d \theta} \operatorname{Re}[s(\theta)]\right|_{\theta=\tilde{\theta}, s=i \nu^{\star}}>0
$$

\footnotetext{
${ }^{19}$ Invoking the properties of the first and second derivative of $f_{1}$ with respect to $\nu^{\star} \theta$ it can also be demonstrated that $\theta^{\star}$ is the only $\theta$ in the interval $\left(0, \pi / \nu^{\star}\right)$ bringing about $f_{1}\left(\nu^{\star}, \nu^{\star} \theta\right)=a_{0}$.

${ }^{20}$ Equation (27) is stated as Lemma 1 in Forde and Nelson (2004, p. 276). However, their proof only appears to establish that the derivative of the real part of the root is nonzero (thus ruling out a degeneracy), while positivity follows from the context according to which all of the roots are assumed to have negative real parts when $\theta$ is less than the bifurcation value.
} 
Together with the stability statement for sufficiently short lags $\theta$, the inequality establishes that all roots of $(20)$ have negative real parts if $0<\theta<\theta^{\star}\left(\theta^{\star}\right.$ being the smallest $\left.\tilde{\theta}\right)$, whereas once a root has entered the positive half-plane it cannot return into the negative half-plane. To sum up, the system is asymptotically stable in the first case, and instability prevails if $\theta>\theta^{\star}$.

To prove the inequality in (27) put $P_{1}(s):=a_{o}+a_{1} s, P_{2}(s):=b_{o}+b_{1} s$ and rewrite the characteristic equation $(20)$ as $Q(s)=P_{1}(s)+P_{2}(s) \exp (-\theta s)=0$. Solving it for $\exp (-\theta s)$ and taking logs, we have

$$
-\theta s=\ln \left[-P_{1}(s) / P_{2}(s)\right]
$$

where $s$ is to be conceived as a function of $\theta$. Differentiating this equation with respect to $\theta$ and evaluating it at $\theta=\tilde{\theta}, s=i \nu=i \nu^{\star}$ gives us (with $P_{k}^{\prime}=d P_{k} / d s, k=1,2$ )

$$
-s-\theta \frac{d s}{d \theta}=\frac{P_{1}^{\prime} P_{2}-P_{1} P_{2}^{\prime}}{P_{1} P_{2}} \frac{d s}{d \theta}=\frac{a_{1} b_{0}-a_{0} b_{1}}{a_{0} b_{0}-\nu^{2} a_{1} b_{1}+i \nu\left(a_{0} b_{1}+a_{1} b_{0}\right)} \frac{d s}{d \theta}
$$

Write the denominator of the ratio on the right-hand side as $\alpha+i \nu \beta$ and note that $\left(a_{1} b_{0}-\right.$ $\left.a_{0} b_{1}\right) \beta=a_{1}^{2} b_{0}^{2}-a_{0}^{2} b_{1}^{2}$. In this way the ratio in (28) can be computed as

$$
\begin{aligned}
\frac{\left(a_{1} b_{0}-a_{0} b_{1}\right)(\alpha-i \nu \beta)}{\alpha^{2}+\nu^{2} \beta^{2}} & =\frac{\left(a_{1} b_{0}-a_{0} b_{1}\right) \alpha-i \nu\left(a_{1}^{2} b_{0}^{2}-a_{0}^{2} b_{1}^{2}\right)}{\alpha^{2}+\nu^{2} \beta^{2}} \\
& =: \quad c-i \nu \delta
\end{aligned}
$$

On this basis eq. (28) can be more compactly formulated as $-i \nu=(\theta+\gamma-i \nu \delta)(d s / d \theta)$. Solving it for $d s / d \theta$ leads to

$$
\frac{d s}{d \theta}=\frac{-i \nu(\theta+\gamma+i \nu \delta)}{(\theta+\gamma)^{2}+\nu^{2} \delta^{2}}=\frac{\nu^{2} \delta-i \nu(\theta+\gamma)}{(\theta+\gamma)^{2}+\nu^{2} \delta^{2}}
$$

It follows that the sign of the real part of $d s / d \theta$ is given by the sign of $\delta$. As this term is proportional to $a_{1}^{2} b_{0}^{2}-a_{0}^{2} b_{1}^{2}$, we can refer to (26) to conclude that $\delta$ is positive. This establishes the positive sign in (27) and so completes the proof.

q.e.d

\section{A.2 Proof of Proposition 5}

From the proof of Proposition 3(c) it follows that if we put $\nu=\nu^{\star}$, the bifurcation value $\theta^{\star}$ of the lag parameter is given as the - unique - value of $\theta$ in the interval $\left(0, \pi / \nu^{\star}\right)$ that satisfies the first equation in (24). Then, observe that the terms $\nu^{\star}, a_{0}, b_{0}$ entering there can 
be factorized as $\nu^{\star}(\mu)=\mu \tilde{\nu}^{\star}, a_{0}(\mu)=\mu \tilde{a}_{0}$ and $b_{0}(\mu)=\mu \tilde{b}_{0}$, where $\tilde{\nu}^{\star}, \tilde{a}_{0}, \tilde{b}_{0}$ are independent of $\mu$ and fixed in the present context. Dividing the said equation by $\mu$, we can say that $\theta^{\star}=\theta^{\star}(\mu)$ is equally determined as the solution of the equation

$$
\tilde{a}_{0}+\tilde{b}_{0} \cos \left(\tilde{\nu}^{\star} \mu \theta\right)+b_{1} \tilde{\nu}^{\star} \sin \left(\tilde{\nu}^{\star} \mu \theta\right)=0
$$

Clearly, if (29) is fulfilled for some $\mu_{1}$ and $\theta_{1}^{\star}$, then the bifurcation lag $\theta^{\star}(\mu)$ of another $\mu$ is given by $\tilde{\nu}^{\star} \mu \theta^{\star}(\mu)=\tilde{\nu}^{\star} \mu_{1} \theta_{1}^{\star}<\pi$; since any other value of $\theta$ satisfying (29) implies $\tilde{\nu}^{\star} \mu \theta>\pi$ and, thus, $\theta>\theta^{\star}(\mu)$.

$$
\text { q.e.d }
$$

\section{A.3 Recursive formulation of the stochastic PBT model}

The history of the $\log$ prices until $p_{t}$, the $\log$ fundamental value $v_{t}$, and the positions $a_{t}^{f}$, $a_{t}^{c}$ of fundamentalists and chartists are predetermined in period $t$. The values of these variables for the next period, besides the inventory of the market maker $a_{t}^{m}$ in the current period, are obtained as follows:

$$
\begin{aligned}
a_{t}^{m} & =-a_{t}^{f}-a_{t}^{c} \\
a_{t+1}^{f} & =\kappa_{f}\left(v_{t}-p_{t}\right) \\
a_{t+1}^{c} & =\kappa_{c}\left(p_{t}-p_{t-\theta}\right) \\
d_{t}^{f} & =a_{t+1}^{f}-a_{t}^{f} \\
d_{t}^{c} & =a_{t+1}^{c}-a_{t}^{c} \\
p_{t+1} & =p_{t}+\left(d_{t}^{f}+d_{t}^{c}-\mu a_{t}^{m}\right) / \lambda \\
v_{t+1} & =\left\{\begin{array}{ll}
v_{t}+\sigma_{v} \delta_{t} & \text { if } t \bmod \tau=0 \\
v_{t} & \text { else }
\end{array} \quad \delta_{t} \sim N(0,1)\right.
\end{aligned}
$$




\section{A.4 Variations of parameters in the stochastic model}

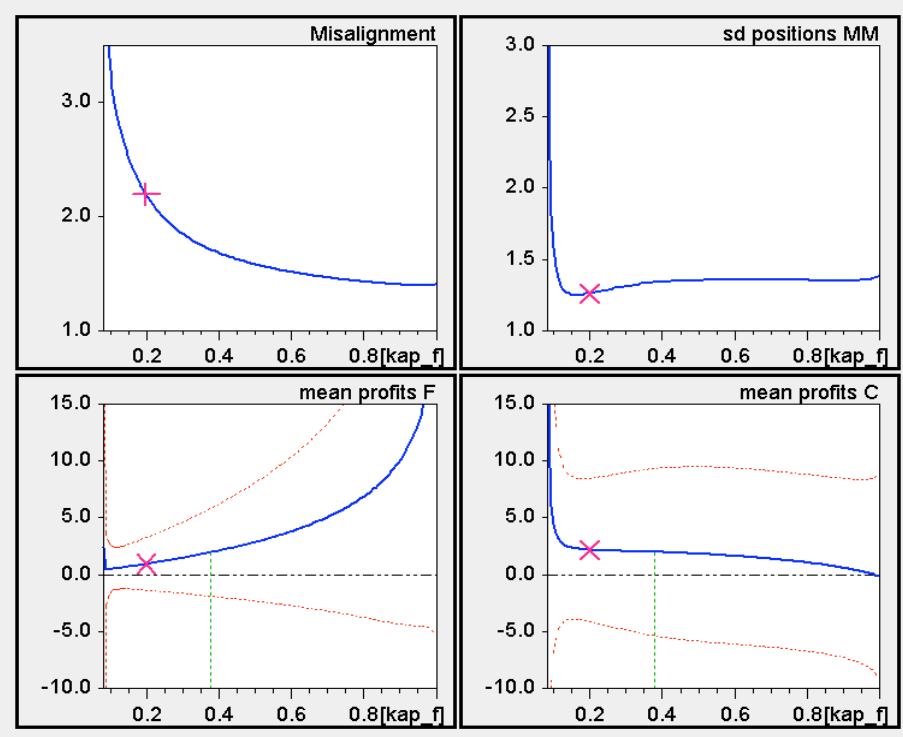

Figure A1: Variations of $\kappa_{f}$.

Note: Fixing the other parameters as given in Table 2, which equally applies to the other figures. See note on Figure 9 for further details.

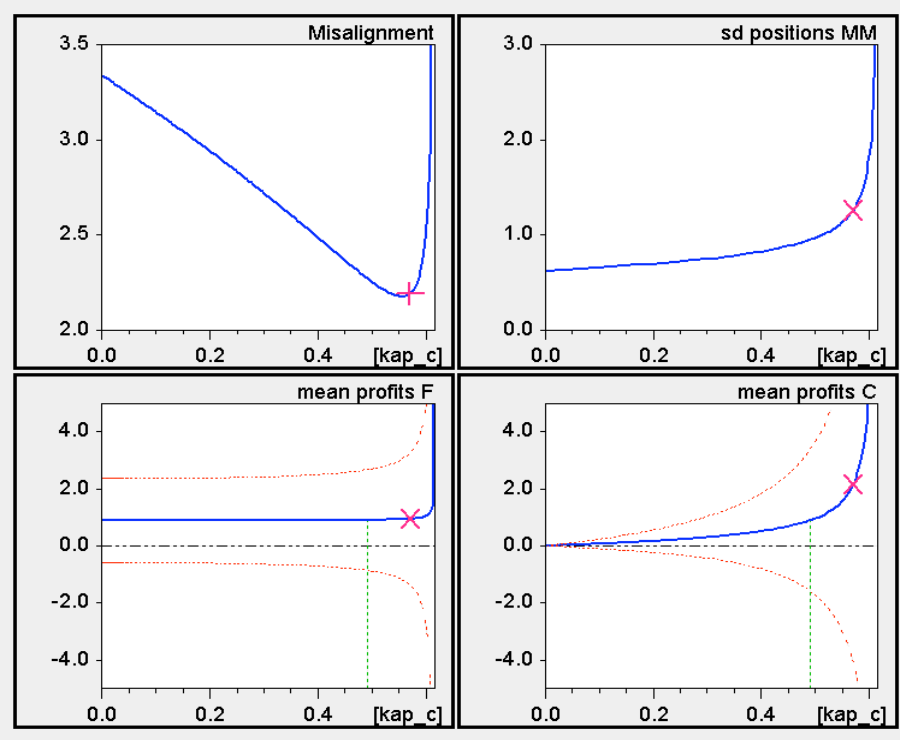

Figure A2: Variations of $\kappa_{c}$. 


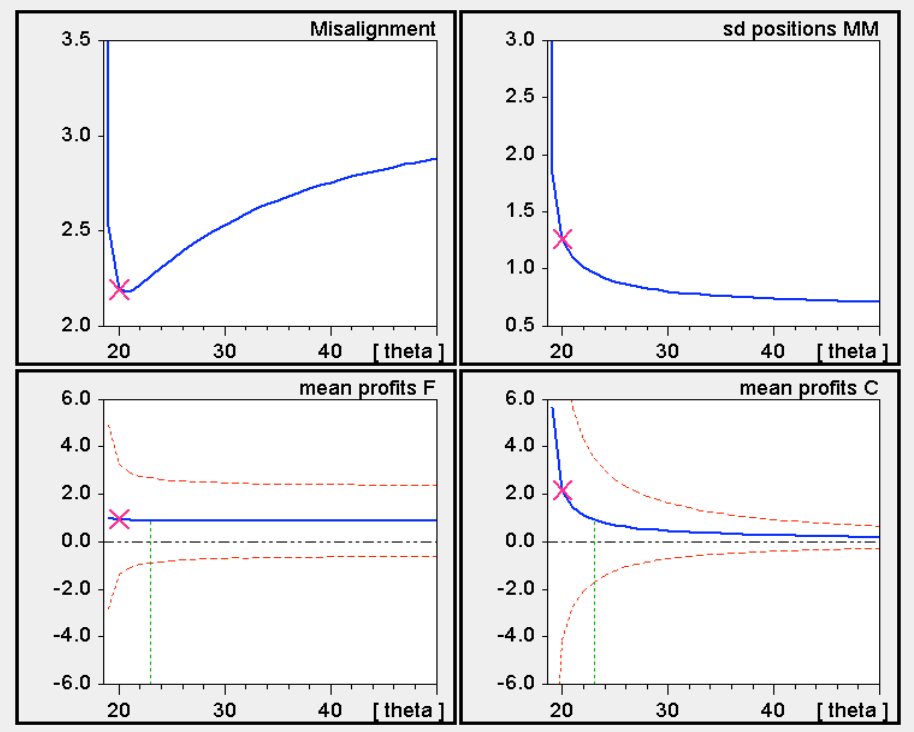

Figure A3: Variations of $\theta$.

Note: Chartist positions specified by eq. (13), with $\tilde{\kappa}_{c}=0.57 \cdot 20=11.40=$ const.

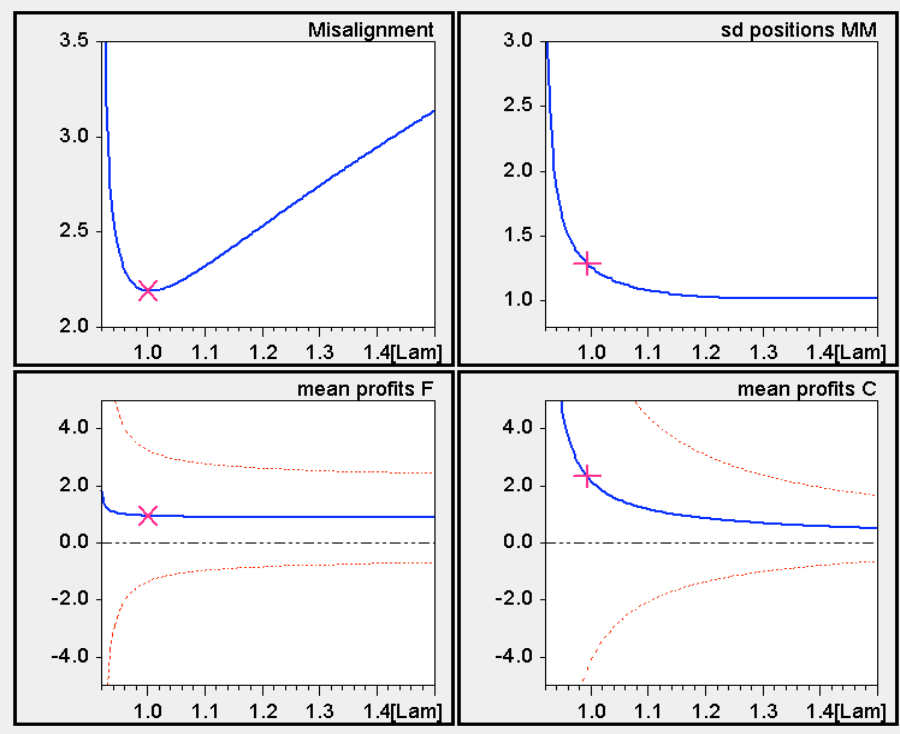

Figure A4: Variations of $\lambda$.

\section{B References}

Arnold, L. (1998), Random Dynamical Systems. New York: Springer. 
BARKER, K. AND WHITESIDES, J.L. (1976), "Stability of neutral equations with constant time delays", NASA Langley Research Center (http://ntrs.nasa.gov./archive/ nasa/casi.ntrs.nasa.gov/19770003358_1977003358.pdf).

Beja, A. And Goldman, M.B. (1980), "On the dynamic behavior of prices in disequilibrium", Journal of Finance, 35, 235-248.

Böhm, V. And Chiarella, C. (2005), "Mean variance preferences, expectations formation, and the dynamics of random asset prices", Mathematical Finance, 15, 61-97.

Bradfield, J. (1979), "A formal dynamic model of market making", Journal of Financial and Quantitative Analysis, 14, 275-291.

Carvalho, R. (2001), "The dynamics of the linear random Farmer model", Instituto Superior Técnico, Lisboa, and University College, London (arXiv: cond-mat/0107150v1).

DAY, R.D. (1997), "Complex dynamics, market mediation and stock price behavior", North American Actuarial Journal, 1:3, 6-23.

FARMER, J.D. (2001), "Toward agent-based models for investment", in Developments in Quantitative Investment Models, AIMR (www . santafe.edu/ jdf/papers/aimr.pdf).

FARmer, J.D. AND Joshi, S. (2002), "The price dynamics of common trading strategies", Journal of Economic Behavior and Organization, 49, 149-171.

Forde, J. And Nelson, P. (2004), "Applications of Sturm sequences to bifurcation analysis of delay differential equation models", Journal of Mathematical Analysis and Applications, 300, 273-284.

Futia, C.A. (1982), "Invariant distributions and the limiting behavior of Markovian economic models", Econometrica, 50, 377-408.

Gandolfo, G. (1997), Economic Dynamics. Berlin: Springer.

Goodwin, R.M. (1951), "The non-linear accelerator and the persistence of business cycles", Econometrica, 19, 1-17.

HAN, Q.-L. (2001), "On delay-dependent stability for neutral delay-differential systems", International Journal of Applied Mathematics and Computer Science, 11, 965-976. 
HE, X.-Z. AND Li, Y. (2007), "Power-law behaviour, heterogeneity, and trend chasing", Journal of Economic Dynamics and Control, 31, 3396-3426.

Hu, G.-D. AND Hu, G.-D. (1996), "Some simple criteria for stability of neutral delaydifferential systems", Applied Mathematics and Computation, 80, 257-271.

Kalecki, M. (1935), "A theory of the business cycle", Econometrica, 3, 327-344.

Markowitz, H. (1959), Portfolio Selection: Efficient Diversification of Investments. New York: John Wiley \& Sons.

Pape, B. (2007), Asset Allocation, Multivariate Position Based Trading, and the Stylized Facts, Doct. Thesis, University of Vaasa.

Schenk-Hoppé, K.R. And Schmalfuss, B. (2001), "Random fixed points in a stochastic Solow growth model", Journal of Mathematical Economics, 36, 19-30.

SETHI, R. (1996), "Endogenous regime switching in financial markets", Structural Change and Economic Dynamics, 7, 99-118.

WesterhofF, F.H. (2003), "Market-maker, inventory control and foreign exchange dynamics", Quantitative Finance, 3, 363-369. 


\section{List of other working papers:}

\section{7}

1. Timur Yusupov and Thomas Lux, The Efficient Market Hypothesis through the Eyes of an Artificial Technical Analyst: An Application of a New Chartist Methodology to High-Frequency Stock Market Data, WP07-13

2. Liu Ruipeng, Di Matteo and Thomas Lux, True and Apparent Scaling: The Proximity of the Markov- Switching Multifractal Model to Long-Range Dependence, WP07-12

3. Thomas Lux, Rational Forecasts or Social Opinion Dynamics? Identification of Interaction Effects in a Business Climate Survey, WP07-11

4. Thomas Lux, Collective Opinion Formation in a Business Climate Survey, WP07-10

5. Thomas Lux, Application of Statistical Physics in Finance and Economics, WP07-09

6. Reiner Franke, A Prototype Model of Speculative Dynamics With Position-Based Trading, WP07-08

7. Reiner Franke, Estimation of a Microfounded Herding Model On German Survey Expectations, WP07-07

8. Cees Diks and Pietro Dindo, Informational differences and learning in an asset market with boundedly rational agents, WP07-06

9. Markus Demary, Who Do Currency Transaction Taxes Harm More: Short-Term Speculators or Long-Term Investors?, WP07-05

10. Markus Demary, A Heterogenous Agents Model Usable for the Analysis of Currency Transaction Taxes, WP07-04

11. Mikhail Anufriev and Pietro Dindo, Equilibrium Return and Agents' Survival in a Multiperiod Asset Market: Analytic Support of a Simulation Model, WP07-03

12. Simone Alfarano and Michael Milakovic, Should Network Structure Matter in Agent-Based Finance?, WP07-02

13. Simone Alfarano and Reiner Franke, A Simple Asymmetric Herding Model to Distinguish Between Stock and Foreign Exchange Markets, WP07-01

\section{6}

1. Roman Kozhan, Multiple Priors and No-Transaction Region, WP06-24

2. Martin Ellison, Lucio Sarno and Jouko Vilmunen, Caution and Activism? Monetary Policy Strategies in an Open Economy, WP06-23

3. Matteo Marsili and Giacomo Raffaelli, Risk bubbles and market instability, WP06-22

4. Mark Salmon and Christoph Schleicher, Pricing Multivariate Currency Options with Copulas, WP06-21

5. Thomas Lux and Taisei Kaizoji, Forecasting Volatility and Volume in the Tokyo Stock Market: Long Memory, Fractality and Regime Switching, WP06-20

6. Thomas Lux, The Markov-Switching Multifractal Model of Asset Returns: GMM Estimation and Linear Forecasting of Volatility, WP06-19

7. Peter Heemeijer, Cars Hommes, Joep Sonnemans and Jan Tuinstra, Price Stability and Volatility in Markets with Positive and Negative Expectations Feedback: An Experimental Investigation, WP06-18

8. Giacomo Raffaelli and Matteo Marsili, Dynamic instability in a phenomenological model of correlated assets, WP06-17

9. Ginestra Bianconi and Matteo Marsili, Effects of degree correlations on the loop structure of scale free networks, WP06-16

10. Pietro Dindo and Jan Tuinstra, A Behavioral Model for Participation Games with Negative Feedback, WP06-15

11. Ceek Diks and Florian Wagener, A weak bifucation theory for discrete time stochastic dynamical systems, WP06-14

12. Markus Demary, Transaction Taxes, Traders' Behavior and Exchange Rate Risks, WP06-13 
13. Andrea De Martino and Matteo Marsili, Statistical mechanics of socio-economic systems with heterogeneous agents, WP06-12

14. William Brock, Cars Hommes and Florian Wagener, More hedging instruments may destabilize markets, WP06-11

15. Ginwestra Bianconi and Roberto Mulet, On the flexibility of complex systems, WP06-10

16. Ginwestra Bianconi and Matteo Marsili, Effect of degree correlations on the loop structure of scale-free networks, WP06-09

17. Ginwestra Bianconi, Tobias Galla and Matteo Marsili, Effects of Tobin Taxes in Minority Game Markets, WP06-08

18. Ginwestra Bianconi, Andrea De Martino, Felipe Ferreira and Matteo Marsili, Multi-asset minority games, WP06-07

19. Ba Chu, John Knight and Stephen Satchell, Optimal Investment and Asymmetric Risk for a Large Portfolio: A Large Deviations Approach, WP06-06

20. Ba Chu and Soosung Hwang, The Asymptotic Properties of AR(1) Process with the Occasionally Changing AR Coefficient, WP06-05

21. Ba Chu and Soosung Hwang, An Asymptotics of Stationary and Nonstationary AR(1) Processes with Multiple Structural Breaks in Mean, WP06-04

22. Ba Chu, Optimal Long Term Investment in a Jump Diffusion Setting: A Large Deviation Approach, WP06-03

23. Mikhail Anufriev and Gulio Bottazzi, Price and Wealth Dynamics in a Speculative Market with Generic Procedurally Rational Traders, WP06-02

24. Simonae Alfarano, Thomas Lux and Florian Wagner, Empirical Validation of Stochastic Models of Interacting Agents: A "Maximally Skewed" Noise Trader Model?, WP06-01

\section{5}

1. Shaun Bond and Soosung Hwang, Smoothing, Nonsynchronous Appraisal and CrossSectional Aggreagation in Real Estate Price Indices, WP05-17

2. Mark Salmon, Gordon Gemmill and Soosung Hwang, Performance Measurement with Loss Aversion, WP05-16

3. Philippe Curty and Matteo Marsili, Phase coexistence in a forecasting game, WP05-15

4. Matthew Hurd, Mark Salmon and Christoph Schleicher, Using Copulas to Construct Bivariate Foreign Exchange Distributions with an Application to the Sterling Exchange Rate Index (Revised), WP05-14

5. Lucio Sarno, Daniel Thornton and Giorgio Valente, The Empirical Failure of the Expectations Hypothesis of the Term Structure of Bond Yields, WP05-13

6. Lucio Sarno, Ashoka Mody and Mark Taylor, A Cross-Country Financial Accelorator: Evidence from North America and Europe, WP05-12

7. Lucio Sarno, Towards a Solution to the Puzzles in Exchange Rate Economics: Where Do We Stand?, WP05-11

8. James Hodder and Jens Carsten Jackwerth, Incentive Contracts and Hedge Fund Management, WP05-10

9. James Hodder and Jens Carsten Jackwerth, Employee Stock Options: Much More Valuable Than You Thought, WP05-09

10. Gordon Gemmill, Soosung Hwang and Mark Salmon, Performance Measurement with Loss Aversion, WP05-08

11. George Constantinides, Jens Carsten Jackwerth and Stylianos Perrakis, Mispricing of S\&P 500 Index Options, WP05-07

12. Elisa Luciano and Wim Schoutens, A Multivariate Jump-Driven Financial Asset Model, WP0506

13. Cees Diks and Florian Wagener, Equivalence and bifurcations of finite order stochastic processes, WP05-05

14. Devraj Basu and Alexander Stremme, CAY Revisited: Can Optimal Scaling Resurrect the (C)CAPM?, WP05-04

15. Ginwestra Bianconi and Matteo Marsili, Emergence of large cliques in random scale-free networks, WP05-03

16. Simone Alfarano, Thomas Lux and Friedrich Wagner, Time-Variation of Higher Moments in a Financial Market with Heterogeneous Agents: An Analytical Approach, WP05-02

17. Abhay Abhayankar, Devraj Basu and Alexander Stremme, Portfolio Efficiency and Discount Factor Bounds with Conditioning Information: A Unified Approach, WP05-01 
1. Xiaohong Chen, Yanqin Fan and Andrew Patton, Simple Tests for Models of Dependence Between Multiple Financial Time Series, with Applications to U.S. Equity Returns and Exchange Rates, WP04-19

2. Valentina Corradi and Walter Distaso, Testing for One-Factor Models versus Stochastic Volatility Models, WP04-18

3. Valentina Corradi and Walter Distaso, Estimating and Testing Sochastic Volatility Models using Realized Measures, WP04-17

4. Valentina Corradi and Norman Swanson, Predictive Density Accuracy Tests, WP04-16

5. Roel Oomen, Properties of Bias Corrected Realized Variance Under Alternative Sampling Schemes, WP04-15

6. Roel Oomen, Properties of Realized Variance for a Pure Jump Process: Calendar Time Sampling versus Business Time Sampling, WP04-14

7. Richard Clarida, Lucio Sarno, Mark Taylor and Giorgio Valente, The Role of Asymmetries and Regime Shifts in the Term Structure of Interest Rates, WP04-13

8. Lucio Sarno, Daniel Thornton and Giorgio Valente, Federal Funds Rate Prediction, WP04-12

9. Lucio Sarno and Giorgio Valente, Modeling and Forecasting Stock Returns: Exploiting the Futures Market, Regime Shifts and International Spillovers, WP04-11

10. Lucio Sarno and Giorgio Valente, Empirical Exchange Rate Models and Currency Risk: Some Evidence from Density Forecasts, WP04-10

11. Ilias Tsiakas, Periodic Stochastic Volatility and Fat Tails, WP04-09

12. Ilias Tsiakas, Is Seasonal Heteroscedasticity Real? An International Perspective, WP04-08

13. Damin Challet, Andrea De Martino, Matteo Marsili and Isaac Castillo, Minority games with finite score memory, WP04-07

14. Basel Awartani, Valentina Corradi and Walter Distaso, Testing and Modelling Market Microstructure Effects with an Application to the Dow Jones Industrial Average, WP04-06

15. Andrew Patton and Allan Timmermann, Properties of Optimal Forecasts under Asymmetric Loss and Nonlinearity, WP04-05

16. Andrew Patton, Modelling Asymmetric Exchange Rate Dependence, WP04-04

17. Alessio Sancetta, Decoupling and Convergence to Independence with Applications to Functional Limit Theorems, WP04-03

18. Alessio Sancetta, Copula Based Monte Carlo Integration in Financial Problems, WP04-02

19. Abhay Abhayankar, Lucio Sarno and Giorgio Valente, Exchange Rates and Fundamentals: Evidence on the Economic Value of Predictability, WP04-01

\section{2}

1. Paolo Zaffaroni, Gaussian inference on Certain Long-Range Dependent Volatility Models, WP02-12

2. Paolo Zaffaroni, Aggregation and Memory of Models of Changing Volatility, WP02-11

3. Jerry Coakley, Ana-Maria Fuertes and Andrew Wood, Reinterpreting the Real Exchange Rate - Yield Diffential Nexus, WP02-10

4. Gordon Gemmill and Dylan Thomas, Noise Training, Costly Arbitrage and Asset Prices: evidence from closed-end funds, WP02-09

5. Gordon Gemmill, Testing Merton's Model for Credit Spreads on Zero-Coupon Bonds, WP0208

6. George Christodoulakis and Steve Satchell, On th Evolution of Global Style Factors in the MSCI Universe of Assets, WP02-07

7. George Christodoulakis, Sharp Style Analysis in the MSCI Sector Portfolios: A Monte Caro Integration Approach, WP02-06

8. George Christodoulakis, Generating Composite Volatility Forecasts with Random Factor Betas, WP02-05

9. Claudia Riveiro and Nick Webber, Valuing Path Dependent Options in the Variance-Gamma Model by Monte Carlo with a Gamma Bridge, WP02-04

10. Christian Pedersen and Soosung Hwang, On Empirical Risk Measurement with Asymmetric Returns Data, WP02-03

11. Roy Batchelor and Ismail Orgakcioglu, Event-related GARCH: the impact of stock dividends in Turkey, WP02-02 
12. George Albanis and Roy Batchelor, Combining Heterogeneous Classifiers for Stock Selection, WP02-01

\section{1}

1. Soosung Hwang and Steve Satchell, GARCH Model with Cross-sectional Volatility; GARCHX Models, WP01-16

2. Soosung Hwang and Steve Satchell, Tracking Error: Ex-Ante versus Ex-Post Measures, WP01-15

3. Soosung Hwang and Steve Satchell, The Asset Allocation Decision in a Loss Aversion World, WP01-14

4. Soosung Hwang and Mark Salmon, An Analysis of Performance Measures Using Copulae, WP01-13

5. Soosung Hwang and Mark Salmon, A New Measure of Herding and Empirical Evidence, WP01-12

6. Richard Lewin and Steve Satchell, The Derivation of New Model of Equity Duration, WP0111

7. Massimiliano Marcellino and Mark Salmon, Robust Decision Theory and the Lucas Critique, WP01-10

8. Jerry Coakley, Ana-Maria Fuertes and Maria-Teresa Perez, Numerical Issues in Threshold Autoregressive Modelling of Time Series, WP01-09

9. Jerry Coakley, Ana-Maria Fuertes and Ron Smith, Small Sample Properties of Panel Timeseries Estimators with I(1) Errors, WP01-08

10. Jerry Coakley and Ana-Maria Fuertes, The Felsdtein-Horioka Puzzle is Not as Bad as You Think, WP01-07

11. Jerry Coakley and Ana-Maria Fuertes, Rethinking the Forward Premium Puzzle in a Nonlinear Framework, WP01-06

12. George Christodoulakis, Co-Volatility and Correlation Clustering: A Multivariate Correlated ARCH Framework, WP01-05

13. Frank Critchley, Paul Marriott and Mark Salmon, On Preferred Point Geometry in Statistics, WP01-04

14. Eric Bouyé and Nicolas Gaussel and Mark Salmon, Investigating Dynamic Dependence Using Copulae, WP01-03

15. Eric Bouyé, Multivariate Extremes at Work for Portfolio Risk Measurement, WP01-02

16. Erick Bouyé, Vado Durrleman, Ashkan Nikeghbali, Gael Riboulet and Thierry Roncalli, Copulas: an Open Field for Risk Management, WP01-01

\section{0}

1. Soosung Hwang and Steve Satchell, Valuing Information Using Utility Functions, WP00-06

2. Soosung Hwang, Properties of Cross-sectional Volatility, WP00-05

3. Soosung Hwang and Steve Satchell, Calculating the Miss-specification in Beta from Using a Proxy for the Market Portfolio, WP00-04

4. Laun Middleton and Stephen Satchell, Deriving the APT when the Number of Factors is Unknown, WP00-03

5. George A. Christodoulakis and Steve Satchell, Evolving Systems of Financial Returns: AutoRegressive Conditional Beta, WP00-02

6. Christian S. Pedersen and Stephen Satchell, Evaluating the Performance of Nearest Neighbour Algorithms when Forecasting US Industry Returns, WP00-01

\section{9}

1. Yin-Wong Cheung, Menzie Chinn and Ian Marsh, How do UK-Based Foreign Exchange Dealers Think Their Market Operates?, WP99-21

2. Soosung Hwang, John Knight and Stephen Satchell, Forecasting Volatility using LINEX Loss Functions, WP99-20

3. Soosung Hwang and Steve Satchell, Improved Testing for the Efficiency of Asset Pricing Theories in Linear Factor Models, WP99-19

4. Soosung Hwang and Stephen Satchell, The Disappearance of Style in the US Equity Market, WP99-18 
5. Soosung Hwang and Stephen Satchell, Modelling Emerging Market Risk Premia Using Higher Moments, WP99-17

6. Soosung Hwang and Stephen Satchell, Market Risk and the Concept of Fundamental Volatility: Measuring Volatility Across Asset and Derivative Markets and Testing for the Impact of Derivatives Markets on Financial Markets, WP99-16

7. Soosung Hwang, The Effects of Systematic Sampling and Temporal Aggregation on Discrete Time Long Memory Processes and their Finite Sample Properties, WP99-15

8. Ronald MacDonald and Ian Marsh, Currency Spillovers and Tri-Polarity: a Simultaneous Model of the US Dollar, German Mark and Japanese Yen, WP99-14

9. Robert Hillman, Forecasting Inflation with a Non-linear Output Gap Model, WP99-13

10. Robert Hillman and Mark Salmon, From Market Micro-structure to Macro Fundamentals: is there Predictability in the Dollar-Deutsche Mark Exchange Rate?, WP99-12

11. Renzo Avesani, Giampiero Gallo and Mark Salmon, On the Evolution of Credibility and Flexible Exchange Rate Target Zones, WP99-11

12. Paul Marriott and Mark Salmon, An Introduction to Differential Geometry in Econometrics, WP99-10

13. Mark Dixon, Anthony Ledford and Paul Marriott, Finite Sample Inference for Extreme Value Distributions, WP99-09

14. Ian Marsh and David Power, A Panel-Based Investigation into the Relationship Between Stock Prices and Dividends, WP99-08

15. Ian Marsh, An Analysis of the Performance of European Foreign Exchange Forecasters, WP99-07

16. Frank Critchley, Paul Marriott and Mark Salmon, An Elementary Account of Amari's Expected Geometry, WP99-06

17. Demos Tambakis and Anne-Sophie Van Royen, Bootstrap Predictability of Daily Exchange Rates in ARMA Models, WP99-05

18. Christopher Neely and Paul Weller, Technical Analysis and Central Bank Intervention, WP9904

19. Christopher Neely and Paul Weller, Predictability in International Asset Returns: A Reexamination, WP99-03

20. Christopher Neely and Paul Weller, Intraday Technical Trading in the Foreign Exchange Market, WP99-02

21. Anthony Hall, Soosung Hwang and Stephen Satchell, Using Bayesian Variable Selection Methods to Choose Style Factors in Global Stock Return Models, WP99-01

\section{8}

1. Soosung Hwang and Stephen Satchell, Implied Volatility Forecasting: A Compaison of Different Procedures Including Fractionally Integrated Models with Applications to UK Equity Options, WP98-05

2. Roy Batchelor and David Peel, Rationality Testing under Asymmetric Loss, WP98-04

3. Roy Batchelor, Forecasting T-Bill Yields: Accuracy versus Profitability, WP98-03

4. Adam Kurpiel and Thierry Roncalli, Option Hedging with Stochastic Volatility, WP98-02

5. Adam Kurpiel and Thierry Roncalli, Hopscotch Methods for Two State Financial Models, WP98-01 